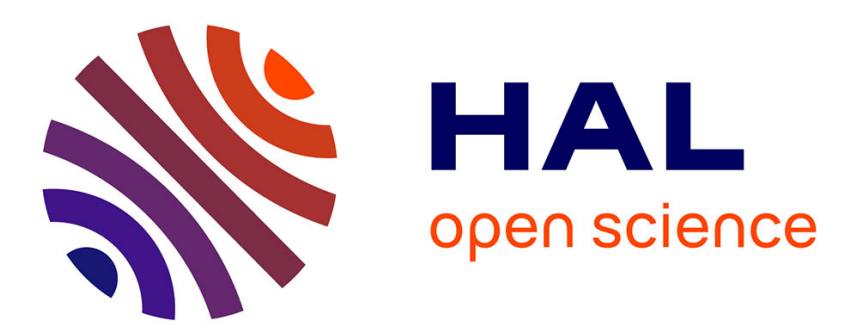

\title{
A unified criterion for the centrifugal instabilities of vortices and swirling jets
}

Paul Billant, Francois Gallaire

\section{To cite this version:}

Paul Billant, Francois Gallaire. A unified criterion for the centrifugal instabilities of vortices and swirling jets. Journal of Fluid Mechanics, 2013, 734, pp.5-35. 10.1017/jfm.2013.460 . hal-00994465

\section{HAL Id: hal-00994465 \\ https://hal-polytechnique.archives-ouvertes.fr/hal-00994465}

Submitted on 26 May 2014

HAL is a multi-disciplinary open access archive for the deposit and dissemination of scientific research documents, whether they are published or not. The documents may come from teaching and research institutions in France or abroad, or from public or private research centers.
L'archive ouverte pluridisciplinaire HAL, est destinée au dépôt et à la diffusion de documents scientifiques de niveau recherche, publiés ou non, émanant des établissements d'enseignement et de recherche français ou étrangers, des laboratoires publics ou privés. 


\title{
A unified criterion for the centrifugal instabilities of vortices and swirling jets
}

\author{
Paul Billant ${ }^{1,} \dagger$ and François Gallaire ${ }^{2}$ \\ ${ }^{1}$ LadHyX, CNRS, École Polytechnique, F-91128 Palaiseau CEDEX, France \\ ${ }^{2}$ Laboratory of Fluid Mechanics and Instabilities, School of Engineering, EPFL, 1015 Lausanne, \\ Switzerland
}

(Received 12 February 2013; revised 14 July 2013; accepted 31 August 2013; first published online 1 October 2013)

Swirling jets and vortices can both be unstable to the centrifugal instability but with a different wavenumber selection: the most unstable perturbations for swirling jets in inviscid fluids have an infinite azimuthal wavenumber, whereas, for vortices, they are axisymmetric but with an infinite axial wavenumber. Accordingly, sufficient condition for instability in inviscid fluids have been derived asymptotically in the limits of large azimuthal wavenumber $m$ for swirling jets (Leibovich and Stewartson, J. Fluid Mech., vol. 126, 1983, pp. 335-356) and large dimensionless axial wavenumber $k$ for vortices (Billant and Gallaire, J. Fluid Mech., vol. 542, 2005, pp. 365-379). In this paper, we derive a unified criterion valid whatever the magnitude of the axial flow by performing an asymptotic analysis for large total wavenumber $\sqrt{k^{2}+m^{2}}$. The new criterion recovers the criterion of Billant and Gallaire when the axial flow is small and the Leibovich and Stewartson criterion when the axial flow is finite and its profile sufficiently different from the angular velocity profile. When the latter condition is not satisfied, it is shown that the accuracy of the Leibovich and Stewartson asymptotics is strongly reduced. The unified criterion is validated by comparisons with numerical stability analyses of various classes of swirling jet profiles. In the case of the Batchelor vortex, it provides accurate predictions over a wider range of axial wavenumbers than the Leibovich-Stewartson criterion. The criterion shows also that a whole range of azimuthal wavenumbers are destabilized as soon as a small axial velocity component is present in a centrifugally unstable vortex.

Key words: instability, jets, vortex flows

\section{Introduction}

The Rayleigh criterion for circular axisymmetric flow with angular velocity $\Omega(r)$ in inviscid and incompressible fluids states that a necessary and sufficient condition for the centrifugal instability is that the square of the circulation $\Gamma=r^{2} \Omega(r)$ decreases with the radius $r$ in some region of the flow (Rayleigh 1917; Synge 1933). Bayly (1988) has shown that this condition remains valid for non-axisymmetric base flows with closed streamlines. The Rayleigh criterion applies only to axisymmetric perturbations but, in the case of axisymmetric flows, Billant \& Gallaire (2005) 
have provided a generalization to non-axisymmetric perturbations by means of a Wentzel-Kramers-Brillouin (WKB) analysis for large axial wavenumber $k$ (made dimensionless by using the typical vortex radius). A sufficient condition for instability of a perturbation of azimuthal wavenumber $m$ is that the real part of the growth rate

$$
\sigma(r)=-\mathrm{i} m \Omega(r)+\sqrt{-\phi(r)},
$$

is positive at the complex radius where $\sigma^{\prime}=0$, where $\phi=\left(1 / r^{3}\right)\left(\Gamma^{2}\right)^{\prime}$ is the Rayleigh discriminant and primes denote differentiation with respect to $r$. In the case of unbounded vortices, this generalized criterion shows that the maximum growth rate of non-axisymmetric disturbances decreases quickly to zero as the azimuthal wavenumber $|m|$ increases. Hence, only a finite band of azimuthal wavenumbers around $m=0$ are centrifugally unstable.

It is well known that the centrifugal instability can also occur on swirling jets, i.e. when there is an axial flow $W(r)$ in addition to the azimuthal motion. Such flows are encountered in many situations: tornadoes, trailing vortices and swirling flows in combustion chambers. Leibovich \& Stewartson (1983) have generalized the Rayleigh criterion to axisymmetric swirling jets by means of an asymptotic analysis for large azimuthal wavenumber $m$ (see also Ludwieg 1960; Emanuel 1984) and shown that a sufficient condition for instability is

$$
\Omega \Omega^{\prime}\left(\Omega^{\prime} \Gamma^{\prime}+W^{\prime 2}\right)<0 .
$$

As pointed out by Eckhoff (1984), this condition is a particular case of a more general condition derived by Eckhoff \& Storesletten (1978) for swirling compressible flows with arbitrary radial distribution of density (see also Sipp et al. 2005; Leblanc \& Le Duc 2005; Di Pierro \& Abid 2010, 2012). When the condition (1.2) is satisfied, the Leibovich-Stewartson asymptotics predict that the maximum growth rate is reached in the limit $|m| \rightarrow \infty$ with a dimensionless axial wavenumber such that $|k / m| \leqslant O(1)$ in agreement with numerical stability results for the Batchelor trailing line vortex (Lessen, Singh \& Paillet 1974; Duck \& Foster 1980; Mayer \& Powell 1992; Delbende \& Rossi 2005).

The azimuthal wavenumbers selected in the cases of swirling jets and pure vortices (i.e. vortices with no axial flow) are therefore completely different. However, none of the aforementioned criteria is able to describe the transition between these two limits: the Leibovich-Stewartson criterion is only valid for large azimuthal wavenumber whereas the generalized Rayleigh criterion (1.1) applies only to vortices without axial flow. The purpose of the present paper is to fill this gap by deriving a criterion valid for any azimuthal wavenumber and any axial flow. Following Billant \& Gallaire (2005) and Leibovich \& Stewartson (1983), we perform a WKB stability analysis of swirling jets for large wavenumber but, instead of considering that either the azimuthal wavenumber or axial wavenumber is large, we assume that $\kappa=\sqrt{m^{2}+k^{2}}$ is large. This quantity $\kappa$ corresponds to the total dimensionless wavenumber at the typical radius of the swirling jet. In this way, we shall be able to describe the stability of swirling jets from the limit $|m| \ll k$ to the opposite limit $|m| \gg k$ when the magnitude of the axial flow varies from zero to a large value compared with the angular motion. Such analysis will provide a criterion that unifies the criteria of Billant \& Gallaire (2005) and Leibovich \& Stewartson (1983). This new criterion will allow us to describe what happens when a small axial flow is added to a centrifugally unstable vortex as encountered in swirling jet experiments or numerical simulations (Sun et al. 2002; Gallaire \& Chomaz 2003; Müller 2007; Müller \& Kleiser 2008). 
The criterion will be also applied to the Batchelor vortex which models trailing line vortices (Batchelor 1964) and whose linear stability has been extensively studied. Its dimensionless azimuthal and axial velocity profiles are defined by

$$
\begin{aligned}
& U_{\theta}(r)=\frac{q}{r}\left(1-e^{-r^{2}}\right), \\
& W(r)=e^{-r^{2}},
\end{aligned}
$$

respectively, where the parameter $q$ controls the strength of the azimuthal motion compared to the axial flow. Lessen et al. (1974) and Mayer \& Powell (1992) have reported that the flow is inviscidly unstable in the range $0<q \lesssim 1.5$. Inviscid instabilities are strongest for $q=0.87$ with a growth rate being 0.46 (Mayer \& Powell 1992). The inviscid stability threshold $q \simeq 1.5$ is close but significantly higher than the value predicted by the Leibovich \& Stewartson (1983) criterion: $q=\sqrt{2}$. However, the asymptotic analysis of Leibovich \& Stewartson (1983) breaks down close to the neutral limits. Stewartson \& Leibovich (1987) and Stewartson \& Capell (1985) have performed refined asymptotic analyses for large azimuthal wavenumber close to the lower and upper neutral limits which are located near $k / m=-q / 2$ and $k / m=-1 / q$, respectively. Near the first limit, the ring modes (modes concentrated in the neighbourhood of a finite radius) considered by Leibovich \& Stewartson (1983) moves close to the vortex axis. At the second limit, the generalized Rayleigh discriminant applying to swirling jets goes to zero and some scaling assumptions in the asymptotic analysis of Leibovich \& Stewartson (1983) break down. Furthermore, by looking when these two limits collide, Stewartson \& Leibovich (1987) have found that the stability threshold is $q=\sqrt{2}(1-1 /(m \sqrt{6}))$ at leading order in $m$. In addition, Stewartson \& Brown (1985) and Heaton (2007a) have both shown that there exists centre modes, i.e. modes concentrated near the axis, in the vicinity of the stability threshold $k / m=-1 / q$ for finite azimuthal wavenumber. These centre modes have been predicted to exist up to $q=2.31$ for $m=-1$ by Stewartson \& Brown (1985). Nevertheless, the maximum growth rate is very small in the range $1.5 \lesssim q<2.31$ and decreases exponentially with $q$ (Heaton 2007a) explaining why the instability threshold has been reported to be $q \simeq 1.5$ by Lessen et al. (1974) and Mayer \& Powell (1992).

There also exist instabilities of a viscous nature (Lessen \& Paillet 1974; Khorrami 1991; Mayer \& Powell 1992; Delbende, Chomaz \& Huerre 1998; Olendraru \& Sellier 2002; Fabre \& Jacquin 2004; Le Dizès \& Fabre 2007; Fabre \& Le Dizès 2008; Le Dizès \& Fabre 2010) whose growth rates are maximum for a finite value of the Reynolds number and decay to zero in the inviscid limit. Their typical growth rate is however much smaller than inviscid instabilities.

Heaton \& Peake (2006) have also discovered algebraic instabilities which are due to the continuous spectrum rather than discrete eigenvalues (i.e. modes). More generally, significant transient growth has been exhibited in swirling jets by Heaton $(2007 \mathrm{~b})$ and Heaton \& Peake (2007), as also described for pure vortices by Antkowiak \& Brancher (2004).

In the present paper, we shall focus on the classical inviscid instabilities with a ring mode structure as considered by Leibovich \& Stewartson (1983) but in the general case of arbitrary axial and angular velocity profiles, i.e. without specializing on the particular case of the Batchelor vortex.

The paper is organized as follows. The stability problem is formulated in $\S 2$ and solved in $\S 3$ for arbitrary velocity profiles by means of an asymptotic analysis for large wavenumbers. Some particular limits which merit further study are investigated 
in $\S 4$ : in $\S 4.1$, the leading orders of the asymptotics of Leibovich \& Stewartson (1983) are retrieved when the axial flow is finite and the shapes of the axial and angular velocity profiles are sufficiently different. The alternative case of similar axial and angular velocity profiles is discussed in $\S 4.2$. The particular cases of uniform and slowly varying angular and axial velocity profiles considered by Leblanc \& Le Duc (2005) and Di Pierro \& Abid (2010), respectively, are treated in $\S 4.3$. Finally, in $\S 4.4$, it is shown that the asymptotic results of Billant \& Gallaire (2005) are recovered in the limit of a small axial flow. The condition determining the maximum growth rate for arbitrary velocity profiles is discussed in $\S 5$. In $\S 6$, the asymptotic formulae are tested against numerical stability results for the Batchelor vortex $(\S 6.1)$ and the Carton $\&$ McWilliams vortex with an axial flow (§ 6.2).

\section{Stability equation}

We consider as a basic flow a swirling jet with velocity components $\left[0, U_{\theta}(r), W(r)\right]$ in cylindrical coordinates $(r, \theta, z)$ in an inviscid and incompressible fluid. Henceforth, we assume that the velocity and length scales are scaled by the maximum norm of the velocity and by the typical radius of the swirling jet, respectively. We subject this basic flow to infinitesimal three-dimensional perturbations of velocity $\hat{\boldsymbol{u}}=\left[\hat{u}_{r}, \hat{u}_{\theta}, \hat{u}_{z}\right]$ and pressure $\hat{p}$ written in the form

$$
[\hat{\boldsymbol{u}}, \hat{p}]=[\boldsymbol{u}(r), p(r)] \mathrm{e}^{\sigma t+\mathrm{i} k z+\mathrm{i} m \theta}+\text { c.c., }
$$

where $\sigma$ is the complex growth rate, $k$ the axial wavenumber, $m$ the azimuthal wavenumber and c.c. denotes the complex conjugate. Without loss of generality, we shall assume that the axial flow on the axis and the axial wavenumber $k$ are positive, whereas the azimuthal wavenumber $m$ can be either sign. Owing to the symmetry $\left(U_{\theta}, m\right) \rightarrow\left(-U_{\theta},-m\right)$, there is also no loss of generality in taking the angular velocity at $r=0$ positive.

The linearized equations of motion for the perturbations can be expressed in terms of a single equation for $\psi=u_{r} \sqrt{r} / Q$ where $Q^{2}=k^{2}+m^{2} / r^{2}$ (Howard \& Gupta 1962; Leibovich \& Stewartson 1983; Stewartson \& Capell 1985)

$$
\frac{\mathrm{d}^{2} \psi}{\mathrm{d} r^{2}}=\tilde{B} \psi
$$

with

$$
\tilde{B}(r)=Q^{2}\left(1+\frac{\Phi}{S^{2}}\right)+\frac{\mathrm{i}}{S}\left(\frac{m}{r^{2}}\left(r \zeta^{\prime}-\frac{\Phi}{\Omega}\right)+k r\left(\frac{W^{\prime}}{r}\right)^{\prime}\right)-\frac{m^{2}\left(Q^{2}+3 k^{2}\right)}{r^{4} Q^{4}}+\frac{3}{4 r^{2}},
$$

where

$$
\Phi=\frac{1}{Q^{2}}\left(k^{2} \phi-2 m k \Omega \frac{W^{\prime}}{r}\right),
$$

is the generalized Rayleigh discriminant and $\zeta=(1 / r)\left(r U_{\theta}\right)^{\prime}$ is the axial vorticity of the basic state, $\phi=2 \Omega \zeta$ is the Rayleigh discriminant, $S=\sigma+\mathrm{i} k W+\mathrm{i} m \Omega$ is the Doppler shifted growth rate and primes denote differentiation with respect to $r$. The boundary conditions are $\psi \rightarrow 0$ as $r \rightarrow \infty$ and as $r \rightarrow 0$ whatever $m$ (Leibovich \& Stewartson 1983). 


\section{WKB stability analysis}

\subsection{Presentation of the asymptotic problem}

We shall solve (2.2) by means of an asymptotic analysis for large wavenumber following Leibovich \& Stewartson (1983) and Billant \& Gallaire (2005). Leibovich \& Stewartson (1983) have assumed that the magnitude of the azimuthal wavenumber $m$ is large and their results show that the unstable axial wavenumbers $k$ lie in the range $q / 2<k /|m|<1 / q$ depending on the swirl parameter $q$. However, these scaling laws apply specifically to the Batchelor vortex which is centrifugally unstable only when the order of magnitude of the azimuthal flow is typically smaller than the axial flow, i.e. in the range $0<O(|\Omega|)<O(|W|)$. In contrast, Billant \& Gallaire (2005) have assumed for pure vortices that $k$ is large and they found that centrifugally unstable perturbations have a finite azimuthal wavenumber $m$. In both cases, one wavenumber is large but it is not the same.

For arbitrary magnitudes of the axial and azimuthal flows, it is convenient to consider the total wavenumber vector $\boldsymbol{Q}=m / r \boldsymbol{e}_{\theta}+k \boldsymbol{e}_{z}$ (Stewartson \& Leibovich 1987) and to assume that its norm is large: $|\boldsymbol{Q}|=Q \gg 1$. Assuming that $r$ is neither too small nor too large, the latter assumption is equivalent to consider that the total dimensionless wavenumber at the typical radius $r=1$ is large: $\kappa=\sqrt{k^{2}+m^{2}} \gg 1$. The use of the quantity $\kappa$ is more convenient than $Q$ since it is independent of $r$. We also denote $\alpha$, the angle of the wavevector $\kappa$ with respect to the vortex axis such that $k=\kappa \cos \alpha$ and $m=\kappa \sin \alpha$. Hence, when $|\alpha|$ varies from 0 to $\pi / 2$, we can switch continuously from the limit of a large axial wavenumber to the limit of a large azimuthal wavenumber. With these definitions, the function $\tilde{B}$ can be rewritten

$$
\tilde{B}=\kappa^{2} B(r)=\kappa^{2}\left(B_{0}(r)+\frac{1}{\kappa} B_{1}(r)+\frac{1}{\kappa^{2}} B_{2}(r)\right),
$$

where

$$
B_{0}=f\left(1+\frac{\Phi}{S^{2}}\right), \quad B_{1}=\frac{H}{S}, \quad B_{2}=-\frac{\sin \alpha^{2}\left(f+3 \cos \alpha^{2}\right)}{r^{4} f^{2}}+\frac{3}{4 r^{2}},
$$

with

$$
\Phi=\frac{\cos \alpha}{f}\left(\phi \cos \alpha-2 \sin \alpha \Omega \frac{W^{\prime}}{r}\right)
$$

and

$$
H=\frac{\mathrm{i} \sin \alpha}{r^{2}}\left(r \zeta^{\prime}-\frac{\Phi}{\Omega}\right)+\mathrm{i} \cos \alpha r\left(\frac{W^{\prime}}{r}\right)^{\prime}, \quad f=\cos \alpha^{2}+\frac{\sin \alpha^{2}}{r^{2}} .
$$

For clarity, we first consider that the dimensionless axial and angular velocities of the base flow are both of order unity. The limits of a slowly rotating jet $[O(\Omega) \ll 1, O(W)=1]$ and a vortex with a small axial flow $[O(\Omega)=1, O(W) \ll 1]$ will be discussed later. When $\Omega=O(1)$ and $W=O(1)$, we see that the functions $f$, $\Phi, H$ and $B_{2}$ are of order unity regardless of $\alpha$ provided that $r=O(1)$. The Doppler shifted growth rate reads $S=\sigma+\mathrm{i} \kappa \Lambda$ with the Doppler shift function

$$
\Lambda=\cos \alpha W+\sin \alpha \Omega \text {. }
$$

Thus, $S$ is generally of order $\kappa$. However, following Leibovich \& Stewartson (1983), we shall assume that the large term $\kappa \Lambda$ is compensated for by the growth rate such 
that we have $S=O(1)$ in a certain range of radius. It follows that $B_{0}$ and $B_{1}$ are also of order unity under the above hypotheses so that $\tilde{B}=O\left(\kappa^{2}\right)$ at leading order.

This scaling of the function $\tilde{B}$ seems to indicate that (2.2) could be solved by means of a complex WKB analysis for large $\kappa$ as done by Billant \& Gallaire (2005) in the case of a pure vortex $(W=0)$. However, the standard WKB approach based on connection formula around simple turning point (Bender \& Orszag 1978) cannot be used directly when there is an axial flow because of the presence of the large term $i \kappa \Lambda$ in the Doppler shifted growth rate $S$. Indeed, even if it is assumed that $S=O(1)$, the derivatives of $S$ are large when $W \neq 0$. Thus, we have

$$
B^{\prime} \sim B_{0}^{\prime}=f^{\prime}\left(1+\frac{\Phi}{S^{2}}\right)+f\left(\frac{\Phi^{\prime}}{S^{2}}-2 \mathrm{i} \kappa \Lambda^{\prime} \frac{\Phi}{S^{3}}\right),
$$

so that $B^{\prime}$ is large: $B^{\prime}(r)=O\left(\kappa \Lambda^{\prime}\right)$ at leading order. Similarly, we have $B^{\prime \prime}(r)=$ $O\left(\kappa^{2} \Lambda^{\prime 2}\right)$ at leading order. This means that the function $B$ varies rapidly with $r$ so that the large parameter $\kappa$ can be eliminated from (2.2) by defining a new variable $u=\kappa r$. Thereby, there is no longer a large parameter justifying the use of the WKB method.

As shown by Leibovich \& Stewartson (1983), this difficulty can be overcome by assuming from the outset that there exist two turning points $r_{1}$ and $r_{2}$ (i.e. $B\left(r_{1}\right)=B\left(r_{2}\right)=0$ ) that are very close for large wavenumber. This implies that there is in between a stationary point $r^{*}$ such that

$$
B^{\prime}\left(r^{*}\right)=0 .
$$

As seen from (3.6), the latter condition can be satisfied only if

$$
\Lambda^{\prime}\left(r^{*}\right) \equiv \cos \alpha W^{\prime}\left(r^{*}\right)+\sin \alpha \Omega^{\prime}\left(r^{*}\right)=O(1 / \kappa),
$$

where we have still assumed that $\Phi\left(r^{*}\right), f\left(r^{*}\right)$ and $S\left(r^{*}\right)$ are of order unity. This relation implies that $\tan \alpha=-W^{\prime}\left(r^{*}\right) / \Omega^{\prime}\left(r^{*}\right)$ at leading order indicating that the wavenumber vector $\boldsymbol{Q}$ is almost parallel to the direction $-W^{\prime} \boldsymbol{e}_{\theta}+r \Omega^{\prime} \boldsymbol{e}_{z}$ along which the strain rate is zero at $r^{*}$ (Emanuel 1984; Stewartson \& Leibovich 1987).

When $\Omega=O(1)$ and $W=O(1)$, the relation (3.8) implies that the angle $|\alpha|$ will be generally different from 0 or $\pi / 2$ so that both the axial wavenumber and the azimuthal wavenumber will be large. However, in the limit of a slowly rotating jet $[O(\Omega) \ll 1, O(W)=1]$, we have $|\alpha| \rightarrow \pi / 2$ so that the axial wavenumber $k$ will be small compared with the azimuthal wavenumber $m$. This limit is encountered for the Batchelor vortex (1.3) when the swirl parameter $q$ is small. In contrast, in the opposite limit of a centrifugally unstable vortex with a small axial flow $[O(\Omega)=1, O(W) \ll 1],(3.8)$ implies $\alpha \rightarrow 0$ meaning that the azimuthal wavenumber $m$ will be small compared with the axial wavenumber $k$.

These two limiting cases are encompassed by assuming that the total wavenumber $\kappa=\sqrt{k^{2}+m^{2}}$ is large whereas $\alpha$ is arbitrary. Nevertheless, we can remark that the neglected terms in (3.8) will have to be taken into account when $\Omega=O(1 / \kappa)$ or $W=O(1 / \kappa)$, i.e. when the magnitude of the axial or azimuthal flow is very small. In these two limits, we have $\Lambda=O(1 / \kappa)$ but it is shown in appendix A that the order of magnitude of the function $B$ remains of order unity at leading order.

When the condition (3.7) is satisfied, equation (2.2) can be written in the neighbourhood of $r^{*}$ :

$$
\frac{\mathrm{d}^{2} \psi}{\mathrm{d} r^{2}}=\kappa^{2} \psi\left[B\left(r^{*}\right)+B^{\prime \prime}\left(r^{*}\right) \frac{\left(r-r^{*}\right)^{2}}{2}\right] .
$$


The higher-order terms of this Taylor expansion, i.e. $B^{\prime \prime \prime}\left(r^{*}\right)\left(r-r^{*}\right)^{3}, B^{i v}\left(r^{*}\right)\left(r-r^{*}\right)^{4}$ and so on, are negligible provided that

$$
\left|r-r^{*}\right| \ll \sqrt{\frac{B^{\prime \prime}\left(r^{*}\right)}{B^{i v}\left(r^{*}\right)}} \sim \frac{1}{\sqrt{\kappa \Lambda^{\prime \prime}\left(r^{*}\right)}},
$$

since $B^{\prime \prime \prime}\left(r^{*}\right)=O\left(\kappa \Lambda^{\prime \prime}, \kappa \Lambda^{\prime \prime \prime}\right)$ and $B^{i v}\left(r^{*}\right)=O\left(\kappa^{2} \Lambda^{\prime \prime 2}\right)$ at leading order.

In turn, the WKB approximations are valid when the following condition is satisfied (Schiff 1968)

$$
\left|\frac{B^{\prime}}{\kappa B^{3 / 2}}\right| \ll 1
$$

Assuming (3.7), this condition is fulfilled when

$$
\left|r-r^{*}\right| \gg \frac{1}{\kappa^{3 / 4} \Lambda^{\prime \prime}\left(r^{*}\right)^{1 / 4}},
$$

i.e. when $r$ is sufficiently far from the turning points $r_{1}=r^{*}-\sqrt{-2 B\left(r^{*}\right) / B^{\prime \prime}\left(r^{*}\right)}$ and $r_{2}=r^{*}+\sqrt{-2 B\left(r^{*}\right) / B^{\prime \prime}\left(r^{*}\right)}$. Comparing the regions of validity of the local solution (3.10) and of the WKB approximations (3.12), we see that there is an overlap region: $\kappa^{-3 / 4} \Lambda^{\prime \prime}\left(r^{*}\right)^{-1 / 4} \ll\left|r-r^{*}\right| \ll \kappa^{-1 / 2} \Lambda^{\prime \prime}\left(r^{*}\right)^{-1 / 2}$ provided that $\Lambda^{\prime \prime}\left(r^{*}\right) \ll \kappa$. The latter condition is always satisfied so that the local solutions of (3.9) can be matched rigourously to WKB approximations in the overlap region.

We can also check that the hypothesis $S(r)=O(1)$ is valid throughout the overlap domain when (3.8) is fulfilled. In addition, the condition (3.11) remains satisfied even for large $\left|r-r^{*}\right|$ since when $\left|r-r^{*}\right| \gg 1 / \sqrt{\kappa \Lambda^{\prime \prime}\left(r^{*}\right)}$, we have $S(r) \gg S\left(r^{*}\right)$ so that $B(r) \approx f(r)=O(1)$ and $B^{\prime}(r)=O(1)$. Hence, the WKB approach is fully justified when a stationary point exists.

The solutions of the local equation (3.9) are parabolic cylinder functions (Bender \& Orszag 1978). These solutions decay on the real $r$-axis as $\kappa^{3 / 4}\left|r-r^{*}\right| \rightarrow \infty$ and can be matched to decaying WKB approximations only if

$$
\kappa \frac{B\left(r^{*}\right)}{\sqrt{B^{\prime \prime}\left(r^{*}\right)}}=-\frac{2 n+1}{\sqrt{2}}+O\left(\sqrt{\frac{\Lambda^{\prime \prime}\left(r^{*}\right)}{\kappa}}\right),
$$

where $n$ is a non-negative integer. The solutions of (3.9) are then

$$
\psi=A H e_{n}(x) \exp \left(-\frac{x^{2}}{4}\right) \quad \text { where } x=2^{1 / 4} \kappa^{1 / 2} B^{\prime \prime}\left(r^{*}\right)^{1 / 4}\left(r-r^{*}\right)
$$

where $A$ is a constant and $\mathrm{He}_{n}(x)$ are Hermite polynomials (Bender \& Orszag 1978). The matching WKB approximations are given in appendix B and compared with the exact eigensolutions for two examples.

Therefore, we conclude that the condition (3.13) holds whatever the magnitude of the axial flow. This relation is indeed identical to the relations derived by Billant \& Gallaire (2005) for pure vortices and by Leibovich \& Stewartson (1983) for swirling jets. Leibovich \& Stewartson (1983) have carried out further their asymptotic analysis to higher orders by considering also the next-order terms of the local equation (3.9). Here, we shall only consider the first-order equation (3.9) since the resulting condition (3.13) yields already satisfactory predictions. 


\subsection{Further expansion of the conditions (3.7) and (3.13)}

The differences between swirling jets and pure vortices are all contained in (3.7) and (3.13) through the function $B$. The most important difference concerns the Doppler shift function $\Lambda$. This function is of order unity when $\Omega=O(1)$ and $W=O(1)$ while it is $O(1 / \kappa)$ when the axial flow is small $W=O(1 / \kappa)$. Furthermore, since $B^{\prime \prime}\left(r^{*}\right)=O\left(\kappa \Lambda^{\prime \prime}\right)$ at leading order, the relation (3.13) implies that $B\left(r^{*}\right)$ is of order $O(1 / \sqrt{\kappa})$ when $\Lambda^{\prime \prime}=O(1)$ while $B\left(r^{*}\right)=O(1 / \kappa)$ when the axial flow is small so that $\Lambda^{\prime \prime} \leqslant O(1 / \kappa)$.

Two distinct asymptotic expansions could be performed for these two cases since the variables scale differently. However, we shall see that it is possible to expand further (3.7) and (3.13) with the wavenumber $\kappa$ and to obtain an explicit expression for the growth rate which is valid whatever the magnitude of $\Lambda$, i.e. both for swirling jets and pure vortices. Indeed, since the conditions (3.7) and (3.13) reduce at leading order to

$$
B_{0}^{\prime}\left(r^{*}\right)=0, \quad B_{0}\left(r^{*}\right)=0,
$$

whatever the order of magnitude of $\Lambda^{\prime \prime}$, it is possible to expand the radius $r^{*}$ and growth rate $\sigma$ in the form

$$
r^{*}=r_{0}+\delta r+\cdots, \quad \sigma=\sigma_{0}+\delta \sigma+\cdots,
$$

where $\left(r_{0}, \sigma_{0}\right)$ will correspond to the solutions of (3.15) while $(\delta r, \delta \sigma)$ will be the next-order corrections with $\delta r \ll r_{0}$ and $\delta \sigma \ll \sigma_{0}$. The scaling with $\kappa$ of these corrections are not specified at this stage because they will depend on $O\left(\Lambda^{\prime \prime}\right)$. For clarity, the dependence of the functions $B_{i}(r)$ with respect to the growth rate $\sigma$ is made explicit by writing $B_{i}(r) \equiv B_{i}(r, \sigma)$. By inserting the decomposition (3.1) and the expansion (3.16), (3.7) becomes at leading orders

$$
\frac{\partial B_{0}}{\partial r}\left(r_{0}, \sigma_{0}\right)+\delta r \frac{\partial^{2} B_{0}}{\partial r^{2}}\left(r_{0}, \sigma_{0}\right)+\delta \sigma \frac{\partial^{2} B_{0}}{\partial r \partial \sigma}\left(r_{0}, \sigma_{0}\right)+\frac{1}{\kappa} \frac{\partial B_{1}}{\partial r}\left(r_{0}, \sigma_{0}\right)+\cdots=0,
$$

and (3.13) yields

$$
\begin{aligned}
& B_{0}\left(r_{0}, \sigma_{0}\right)+\delta r \frac{\partial B_{0}}{\partial r}\left(r_{0}, \sigma_{0}\right)+\delta \sigma \frac{\partial B_{0}}{\partial \sigma}\left(r_{0}, \sigma_{0}\right)+\frac{B_{1}\left(r_{0}, \sigma_{0}\right)}{\kappa}+\frac{\delta r}{\kappa} \frac{\partial B_{1}}{\partial r}\left(r_{0}, \sigma_{0}\right) \\
& +\frac{\delta \sigma}{\kappa} \frac{\partial B_{1}}{\partial \sigma}\left(r_{0}, \sigma_{0}\right)=-\frac{2 n+1}{\sqrt{2} \kappa} \sqrt{\frac{\partial^{2} B_{0}}{\partial r^{2}}\left(r_{0}, \sigma_{0}\right)+\frac{1}{\kappa} \frac{\partial^{2} B_{1}}{\partial r^{2}}\left(r_{0}, \sigma_{0}\right)}+O\left(\frac{\Lambda^{\prime \prime}\left(r_{0}\right)}{\kappa}\right) .
\end{aligned}
$$

\subsubsection{Leading-order problem}

At leading order, the relations (3.17) and (3.18) reduce to (3.15), i.e.

$$
\begin{gathered}
\frac{\partial B_{0}}{\partial r}\left(r_{0}, \sigma_{0}\right)=0, \\
B_{0}\left(r_{0}, \sigma_{0}\right)=0 .
\end{gathered}
$$

Equation (3.19b) implies directly

$$
\sigma_{0}=-\mathrm{i} \kappa \Lambda\left(r_{0}\right)+\sqrt{-\Phi\left(r_{0}\right)},
$$

while $(3.19 a)$ is satisfied if

$$
\frac{\partial \sigma_{0}}{\partial r_{0}}=0
$$


that is,

$$
\Lambda^{\prime}\left(r_{0}\right)=\mathrm{i} \frac{\Phi^{\prime}\left(r_{0}\right)}{2 \kappa \sqrt{-\Phi\left(r_{0}\right)}} .
$$

Since $\kappa \gg 1$ whereas $\Phi=O(1)$, the latter equation reduces at leading order to

$$
\Lambda^{\prime}\left(r_{0}\right)=0 \text {. }
$$

The leading-order relations (3.20) and (3.23) correspond exactly to those found by Leibovich \& Stewartson (1983). However, in contrast to all of the hypotheses employed so far, equation (3.23) is not valid for all types of swirling jet. First, it is not valid for vortices with a weak axial flow $[W=O(1 / \kappa), O(\Omega)=1]$, because, as discussed previously $\Lambda(r)$ and all of its derivatives are then of order $O(1 / \kappa)$. In the latter case, it is not possible to simplify (3.22) further.

Furthermore, even for swirling jets with $O(W)=O(\Omega)=1$, we shall see in $\S 4.2$ that the approximation (3.23) is not valid when the shapes of the axial and angular velocity profiles are similar.

Therefore, in the purpose of deriving a general relation encompassing pure vortices and all types of swirling jet, we keep (3.22) under this form for the moment. Since the term in the right-hand side of (3.22) is either smaller or comparable with the left-hand side, we emphasize that it may be unnecessary to keep this term at leading order in some cases but, in any case, no error is introduced.

\subsubsection{Next-order problem}

We now proceed to the next order of (3.17) and (3.18) in order to determine the first-order corrections $(\delta r, \delta \sigma)$. The correction $\delta r$ can be found from (3.17). Its order of magnitude is $\delta r=O\left(\delta \sigma /\left(\kappa \Lambda^{\prime \prime}\right), \partial B_{1} / \partial r /\left(\kappa^{2} \Lambda^{\prime \prime}\right)\right)$ but, in fact, this correction is not needed to determine the growth rate correction $\delta \sigma$. This correction can be indeed obtained explicitly and directly from (3.18)

$$
\delta \sigma=-\frac{1}{\kappa}\left(\frac{\partial B_{0}}{\partial \sigma}\left(r_{0}, \sigma_{0}\right)\right)^{-1}\left(\frac{2 n+1}{\sqrt{2}} \sqrt{\frac{\partial^{2} B_{0}}{\partial r^{2}}\left(r_{0}, \sigma_{0}\right)}+B_{1}\left(r_{0}, \sigma_{0}\right)\right) .
$$

It is crucial to realize that this formula has been obtained without any particular hypothesis on the magnitude of $\Lambda$ : all of the neglected terms in (3.18) remain small compared with the retained terms as long as $\Lambda^{\prime \prime} \leqslant O(1)$. It is thus valid whatever the magnitudes of the angular and axial velocities.

\subsection{General growth rate formula}

By substituting $B_{0}$ and $B_{1}$ by their expression (3.2a,b) into (3.24) and by using (3.19) and (3.20), we obtain an explicit and general expression for the growth rate:

$$
\begin{aligned}
\sigma= & -\mathrm{i} \kappa \Lambda\left(r_{0}\right)+\sqrt{-\Phi\left(r_{0}\right)} \\
& -\frac{2 n+1}{2 \kappa \sqrt{2 f\left(r_{0}\right)}} \sqrt{\Phi^{\prime \prime}\left(r_{0}\right)-\frac{\Phi^{\prime}\left(r_{0}\right)^{2}}{2 \Phi\left(r_{0}\right)}+2 \mathrm{i} \sqrt{-\Phi\left(r_{0}\right)} \kappa \Lambda^{\prime \prime}\left(r_{0}\right)} \\
& -\frac{H\left(r_{0}\right)}{2 \kappa f\left(r_{0}\right)}+O\left(\frac{\Lambda^{\prime \prime}\left(r_{0}\right)}{\kappa}\right)
\end{aligned}
$$

where we recall that $r_{0}$ is given by (3.22). This formula is the main result of the paper. When going from (3.24) to (3.25), we stress that no term has been neglected 
and the general expressions (3.20) and (3.22) for the leading-order growth rate and radius $\left(\sigma_{0}, r_{0}\right)$ have been used so that $(3.25)$ be the most general. However, we see that (3.25) could be further simplified for swirling jets when $\Lambda^{\prime \prime}$ is of order unity. The term $2 \mathrm{i} \sqrt{-\Phi\left(r_{0}\right)} \kappa \Lambda^{\prime \prime}\left(r_{0}\right)$ in the square root is then dominant compared with the two first terms. Similarly, the last term of (3.25) should be neglected since it is of order $1 / \kappa$ like the neglected higher-order terms. However, these simplifications do not apply for all types of velocity profiles. As mentioned previously, we can have $\Lambda^{\prime \prime} \leqslant O(1 / \kappa)$ for vortices with a small axial flow. In $\S 4.2$, we shall also show that the scaling $\Lambda^{\prime \prime} \leqslant O(1 / \kappa)$ is encountered for swirling jets with similar axial and angular velocity profiles. For this reason, (3.25) is kept under this universal form at this stage.

The formula (3.25) implies that $S\left(r_{0}\right)=\sqrt{-\Phi\left(r_{0}\right)}$ at leading order. Hence, the $a$ priori assumption $S\left(r_{0}\right)=O(1)$ is fulfilled as long as $\Phi\left(r_{0}\right)$ is not too small. In contrast, when $\Phi\left(r_{0}\right)=O\left(1 / \kappa^{2}\right)$, we have $S\left(r_{0}\right)=O(1 / \kappa)$ so that $B_{1} / \kappa \sim B_{0}$. Then, the function $B_{1}$ needs to be taken into account at leading order. This limit is discussed in appendix $\mathrm{C}$.

Another condition of validity is that $r_{0}$ should not be too small, typically $r_{0} \gg 1 / \kappa$ in order that the local solution near the stationary point $r_{0}$ can be connected to the regular solution near the axis $r=0$.

In $\S 4.1$, we first simplify (3.25) and (3.22) in the limit where $\kappa \Lambda^{\prime \prime}\left(r_{0}\right)$ is effectively large. In $\S 4.2$, we discuss the conditions required to have $O\left(\kappa \Lambda^{\prime \prime}\left(r_{0}\right)\right) \gg 1$. In $\S 4.3$, we consider the particular cases of constant or slowly varying axial and angular velocity profiles. Finally, in $\S 4.4$, we simplify (3.25) and (3.22) when the axial flow $W$ is small. As discussed in appendix A, the limit of a slowly rotating jet is equivalent to a standard swirling jet after an ad hoc rescaling of the variables. The growth rate in this limit is thus not particularly different from (3.25).

\section{Analyses in particular limits}

\subsection{Leibovich \& Stewartson (1983) asymptotics}

We now consider that $\Lambda^{\prime \prime}\left(r_{0}\right)$ is of order unity as considered by Leibovich \& Stewartson (1983) and we assume that (3.23) is valid at leading order. Then, equation (3.22) can be solved by expanding $r_{0}$ in the form

$$
r_{0}=r_{00}+\frac{r_{01}}{\kappa}+\cdots
$$

We then retrieve (3.23) at leading order

$$
\Lambda^{\prime}\left(r_{00}\right)=0
$$

and, at first order, we have

$$
r_{01}=\mathrm{i} \frac{\Phi^{\prime}\left(r_{00}\right)}{2 \sqrt{-\Phi\left(r_{00}\right)}} \frac{1}{\Lambda^{\prime \prime}\left(r_{00}\right)} .
$$

Introducing the expansion (4.1) in (3.25) gives

$$
\sigma=-\mathrm{i} \kappa \Lambda\left(r_{00}\right)+\sqrt{-\Phi\left(r_{00}\right)}-\frac{2 n+1}{\sqrt{\kappa}}(1+\mathrm{i}) \frac{\left(-\Phi\left(r_{00}\right)\right)^{1 / 4}}{2 \sqrt{2 f\left(r_{00}\right)}} \sqrt{\Lambda^{\prime \prime}\left(r_{00}\right)}+O\left(\frac{1}{\kappa}\right) .
$$

When applied to the Batchelor vortex (1.3) and expressed in term of the azimuthal wavenumber $m=\kappa \sin \alpha$, the formula (4.4) recovers the first orders of the equations (4.39)-(4.40) given by Leibovich \& Stewartson (1983) (or (6.1)-(6.2) in Stewartson \& Capell (1985) amended for typographical errors). Leibovich \& 
Stewartson (1983) have also computed the next two higher-order terms, i.e. $O\left(\kappa^{-1}\right)$ and $O\left(\kappa^{-3 / 2}\right)$, for the leading unstable mode $n=0$ for the Batchelor vortex. These higher-order terms are given in appendix D.

Leibovich \& Stewartson (1983) have shown that the radius $r_{00}$ is always purely real in the case of the Batchelor vortex. The maximum growth rate is therefore given by the maximum of $\sqrt{-\tilde{\Phi}}$ over real radius $r$ where $\tilde{\Phi}$ is obtained by inserting the relation (4.2) in (3.3):

$$
\tilde{\Phi}(r)=\frac{2 \Omega \Omega^{\prime} r}{\Omega^{\prime 2} r^{2}+W^{\prime 2}}\left(\Omega^{\prime} r \zeta+W^{\prime 2}\right) .
$$

Hence, a sufficient condition for instability is that $\tilde{\Phi}(r)<0$ for some radius $r$, i.e. the condition (1.2) (Leibovich \& Stewartson 1983).

\subsection{Swirling jets with similar axial and angular velocity profiles}

In the previous section, we have seen that the Leibovich \& Stewartson (1983) growth rate is recovered when $\Lambda^{\prime \prime}\left(r_{00}\right)$ is assumed to be of order unity. However, this scaling is not always valid for swirling jets. Indeed, using (4.2), we can write

$$
\Lambda^{\prime \prime}\left(r_{00}\right)=\cos \alpha\left(W^{\prime \prime}\left(r_{00}\right)-\Omega^{\prime \prime}\left(r_{00}\right) \frac{W^{\prime}\left(r_{00}\right)}{\Omega^{\prime}\left(r_{00}\right)}\right) .
$$

This shows directly that if the axial velocity profile is a linear function of the angular velocity profile, i.e. $W(r)=W_{0} \Omega(r)+C$ where $W_{0}$ and $C$ are constants, we have $\Lambda^{\prime \prime}\left(r_{00}\right)=0$. Thus, the expansion (4.1) breaks down since $r_{01}=\infty$ and (4.4) is no longer valid. Such breakdown of the asymptotics of Leibovich \& Stewartson (1983) has been already noted by Leblanc \& Le Duc (2005) in the particular case where $\Lambda$ is uniform in space. This will be further discussed in the next subsection.

In order to determine the conditions required for the asymptotics of Leibovich \& Stewartson (1983) to be valid for varying velocity profiles, it is interesting to consider that the axial velocity profile is slightly different from the angular velocity profile

$$
W(r)=W_{0}[\Omega(r)+\epsilon(r)]+C,
$$

with $\epsilon(r) \ll \Omega(r)$. Then, we have

$$
\Lambda^{\prime \prime}\left(r_{00}\right)=W_{0} \cos \alpha\left[\epsilon^{\prime \prime}\left(r_{00}\right)-\epsilon^{\prime}\left(r_{00}\right) \frac{\Omega^{\prime \prime}\left(r_{00}\right)}{\Omega^{\prime}\left(r_{00}\right)}\right] .
$$

Note that we assume $\Omega^{\prime} \neq 0$ since $\Omega$ is monotonically decreasing with $r$ for most velocity profiles. Equation (4.8) shows that $\kappa \Lambda^{\prime \prime}\left(r_{00}\right) \gg 1$ when $W_{0}=O(1)$ only if

$$
O\left(\epsilon^{\prime}, \epsilon^{\prime \prime}\right) \gg 1 / \kappa \text {. }
$$

It emerges that the expansion (4.1) can be performed only if the shapes of the axial and angular velocity profiles are sufficiently different relative to $1 / \kappa$. The difference $\epsilon$ between the angular and axial velocity profiles is therefore an additional important parameter of the problem. Since $\epsilon$ is non-zero for most velocity profiles, the condition (4.9) will be generally satisfied for sufficiently large wavenumber $\kappa$. Hence, one could think that it is unnecessary to worry about this condition since we are in the framework of the WKB approximation which is also valid for large wavenumber. However, the WKB approximation is known to be very powerful and to provide accurate results for moderate values of $\kappa$ and even down to $\kappa=O(1)$. Therefore, it is likely that the condition (4.9) will put a stronger constraint on the value of $\kappa$ needed 
to obtain accurate results than the WKB approximation. In other words, the expansion (4.1) can deteriorate the excellent accuracy intrinsic to the WKB method.

The determination of the radius $r_{0}$ is completely different when the condition (4.9) is not satisfied. Indeed, inserting the axial velocity profile (4.7) into (3.22) yields

$$
\Phi^{\prime}\left(r_{0}\right)=-2 \mathrm{i} \kappa\left[\left(W_{0} \cos \alpha+\sin \alpha\right) \Omega^{\prime}\left(r_{0}\right)+W_{0} \cos \alpha \epsilon^{\prime}\left(r_{0}\right)\right] \sqrt{-\Phi\left(r_{0}\right)} .
$$

This becomes, at leading order,

$$
\left(W_{0} \cos \alpha+\sin \alpha\right) \Omega^{\prime}\left(r_{0}\right)=0 .
$$

Since $\Omega^{\prime}\left(r_{0}\right) \neq 0$, this relation is satisfied only for a single angle $\alpha_{0}$ such that $\tan \alpha_{0}=-W_{0}$ and the radius $r_{0}$ remains undetermined. If we write $\alpha=\alpha_{0}+\delta \alpha$ with $\delta \alpha=O(1 / \kappa)$, we obtain at the next order

$$
\Phi^{\prime}\left(r_{0}\right)=-2 \mathrm{i} \kappa \cos \alpha_{0}\left[\delta \alpha\left(W_{0}^{2}+1\right) \Omega^{\prime}\left(r_{0}\right)+W_{0} \epsilon^{\prime}\left(r_{0}\right)\right] \sqrt{-\Phi\left(r_{0}\right)} .
$$

This relation allows one to determine $r_{0}$ for a given angle $\alpha_{0}+\delta \alpha$. However, it is not simpler than the original equation (3.22).

These considerations show that the approximation (4.2) is far from being universal and can be used only under certain circumstances. This feature will be confirmed in $\S 6$ since we shall see that the approximation (3.22) is more robust than (4.2) for arbitrary velocity profiles. Even for the Batchelor vortex, equation (3.22) turns out to be valid over a wider range of axial wavenumbers than (4.2).

Finally, we emphasize that all of the retained terms in the formula (3.25) are large compared with the neglected terms when $O\left(\epsilon^{\prime}, \epsilon^{\prime \prime}\right)=1 / \kappa$.

\subsection{Swirling jets with constant or slowly varying angular and axial velocity profiles}

Leblanc \& Le Duc (2005) have considered the asymptotic stability for large wavenumbers of compressible swirling flows with a radial distribution of density, a problem which is more general but not significantly different from the incompressible and homogeneous density case considered herein. As mentioned before, they have shown that the asymptotics of Leibovich \& Stewartson (1983) break down when $\Lambda$ is uniform in space. This occurs when both $\Omega$ and $W$ are uniform, or when $\Omega$ is uniform and $k=0$ or when $W$ is uniform and $m=0$. We can show that (3.25) and (3.22) in these three cases recover formally the formula of Leblanc \& Le Duc (2005) if the density is uniform and the fluid incompressible. Indeed, when $\Lambda=\Lambda_{0}$ is constant, (3.22) reduces to

$$
\Phi^{\prime}\left(r_{0}\right)=0,
$$

while (3.25) becomes

$$
\sigma=-\mathrm{i} \kappa \Lambda_{0}+\sqrt{-\Phi\left(r_{0}\right)}-\frac{2 n+1}{2 \kappa \sqrt{2 f\left(r_{0}\right)}} \sqrt{\Phi^{\prime \prime}\left(r_{0)}\right.}-\frac{H\left(r_{0}\right)}{2 \kappa f\left(r_{0}\right)} .
$$

When the axial and azimuthal velocities are constant, i.e. $\Omega(r)=\Omega_{0}$ and $W(r)=W_{0}$, we have $\Phi\left(r_{0}\right)=4 \Omega_{0}^{2} k^{2} r_{0}^{2} /\left(k^{2} r_{0}^{2}+m^{2}\right)$ and $H\left(r_{0}\right)=-4 \mathrm{i} \Omega_{0} m k^{2} /\left(\kappa\left(m^{2}+k^{2} r_{0}^{2}\right)\right)$. Then, (4.14) is identical to the equation (4.5) of Leblanc \& Le Duc (2005) in the case of an homogeneous and incompressible fluid. Note that there is no instability in this case since $\Phi$ is everywhere positive. When $\Omega(r)=\Omega_{0}$ and $k=0$ or when $W(r)=W_{0}$ and $m=0$, equation (4.14) agrees also formally with the results of Leblanc \& Le Duc (2005) when the density is uniform and the fluid incompressible. 
More recently, Di Pierro \& Abid (2010) have considered variable-density incompressible swirling flows and extended the analysis of Leblanc \& Le Duc (2005) to the case where the axial and angular velocity profiles vary slowly with $r$ such that

$$
\Omega(r)=\Omega_{0}+\frac{1}{\kappa^{3 / 2}} \tilde{\Omega}(r), \quad W(r)=W_{0}+\frac{1}{\kappa^{3 / 2}} \tilde{W}(r),
$$

where $\tilde{\Omega}(r), \tilde{W}(r)$ are assumed to be of order unity. Under this assumption, equation (3.22) becomes

$$
\frac{1}{\kappa^{1 / 2}} \tilde{\Lambda}^{\prime}\left(r_{0}\right)=\mathrm{i} \frac{\Phi^{\prime}\left(r_{0}\right)}{2 \sqrt{-\Phi\left(r_{0}\right)}},
$$

where $\tilde{\Lambda}=\cos \alpha \tilde{W}+\sin \alpha \tilde{\Omega}$. This equation can be solved by expanding the radius $r_{0}$ in the form

$$
r_{0}=r_{00}+\frac{r_{01}}{\kappa^{1 / 2}}+\cdots
$$

giving at first and second orders

$$
\begin{gathered}
\Phi^{\prime}\left(r_{00}\right)=0, \\
r_{01}=-2 \mathrm{i} \tilde{\Lambda}^{\prime}\left(r_{00}\right) \frac{\sqrt{-\Phi\left(r_{00}\right)}}{\Phi^{\prime \prime}\left(r_{00}\right)} .
\end{gathered}
$$

Inserting (4.15) and (4.17) in (3.25) leads to

$$
\begin{aligned}
\sigma= & -\mathrm{i} \kappa \Lambda_{0}+\sqrt{-\Phi\left(r_{00}\right)}-\frac{\mathrm{i}}{\kappa^{1 / 2}} \tilde{\Lambda}\left(r_{00}\right) \\
& -\frac{2 n+1}{2 \kappa \sqrt{2 f\left(r_{00}\right)}} \sqrt{\Phi^{\prime \prime}\left(r_{00}\right)}-\tilde{\Lambda}^{\prime}\left(r_{00}\right)^{2} \frac{\sqrt{-\Phi\left(r_{00}\right)}}{\kappa \Phi^{\prime \prime}\left(r_{00}\right)}-\frac{H\left(r_{00}\right)}{2 \kappa f\left(r_{00}\right)}+O\left(\kappa^{-3 / 2}\right),
\end{aligned}
$$

where $\Lambda_{0}=\cos \alpha W_{0}+\sin \alpha \Omega_{0}$ and $H\left(r_{00}\right)=-4 \mathrm{i} \Omega_{0} \sin \alpha \cos \alpha^{2} /\left(r_{00}^{2} f\left(r_{00}\right)\right)$ at leading order. If we further consider that $\tilde{W}\left(r_{00}\right)=\tilde{\Omega}\left(r_{00}\right)=0$ as assumed by Di Pierro \& Abid (2010), we have $\tilde{\Lambda}\left(r_{00}\right)=0$ and (4.20) recovers the formula (18) of Di Pierro \& Abid (2010) in the limit of an homogeneous fluid. Even if the assumption (4.15) of slowly varying angular and axial velocity profiles does not apply to the Batchelor or Carton \& McWilliams vortices, the formula (4.20) will also be tested for these vortices in $\S 6$.

\subsection{Small axial flow}

When the axial flow is small such that $W=O(1 / \kappa)$ independently of $r$ whereas $\Omega=O(1)$, equation (3.22) shows that $\alpha=O(1 / \kappa)$ so that the azimuthal wavenumber is finite $m=O(1)$ and the Doppler shift function is small $\Lambda=O(1 / \kappa)$. It follows that $\kappa=k+O(1 / k), \Phi(r)=\phi(r)+O\left(1 / k^{2}\right)$ and $f=1+O\left(1 / k^{2}\right)$. Then, the asymptotic growth rate (3.25) reduces to

$$
\begin{aligned}
\sigma= & -\mathrm{i} k W\left(r_{0}\right)-\mathrm{i} m \Omega\left(r_{0}\right)+\sqrt{-\phi\left(r_{0}\right)} \\
& -\frac{2 n+1}{2 \sqrt{2} k} \sqrt{\phi^{\prime \prime}\left(r_{0}\right)-\frac{\phi^{\prime}\left(r_{0}\right)^{2}}{2 \phi\left(r_{0}\right)}+2 \mathrm{i} \sqrt{-\phi\left(r_{0}\right)}\left[k W^{\prime \prime}\left(r_{0}\right)+m \Omega^{\prime \prime}\left(r_{0}\right)\right]} \\
& +O\left(\frac{1}{k^{2}}\right) .
\end{aligned}
$$


The last term of (3.25) is $O\left(1 / k^{2}\right)$ and thereby has been neglected. Equation (3.22) for $r_{0}$ becomes

$$
\phi^{\prime}\left(r_{0}\right)=-2 \mathrm{i}\left(k W^{\prime}\left(r_{0}\right)+m \Omega^{\prime}\left(r_{0}\right)\right) \sqrt{-\phi\left(r_{0}\right)} .
$$

The formulae (4.21) and (4.22) are exactly the same as for pure vortices (Billant $\&$ Gallaire 2005) excepted that the Doppler shift function is $k W+m \Omega$ instead of $m \Omega$.

For pure vortices $(W=0)$, Billant \& Gallaire (2005) have shown that the maximum growth rate for a given azimuthal wavenumber $m$ is reached in the limit $k \rightarrow \infty$ and is $\sigma_{r \max }=\operatorname{Re}\left(-\mathrm{i} m \Omega\left(r_{0}\right)+\sqrt{-\phi\left(r_{0}\right)}\right)$. The growth rate in this limit is maximum for $m=0$ and monotonically decreases as $|m|$ increases up to a finite azimuthal wavenumber cutoff $m_{c}$ which depends on each particular angular velocity profile.

Using these results for pure vortices, a basic understanding of the effect of the additional term $k W$ can be easily obtained by considering the axial velocity profile $W(r)=W_{0} \Omega(r)$. In this simple case, the formulae (4.21) and (4.22) become identical to those for pure vortices except that $m$ is replaced by $m+k W_{0}$. Hence, the stability properties of this swirling jet can be directly deduced from the results of Billant $\&$ Gallaire (2005) for pure vortices. The fact that the maximum growth rate is reached for $m=0$ when $W_{0}=0$ implies that the most amplified axial wavenumber for a given azimuthal wavenumber $m$ will be $k=-m / W_{0}$ for this swirling jet. The associated maximum growth rate will be $\sigma_{\max }=\sqrt{-\phi\left(r_{0}\right)}$ where $\phi^{\prime}\left(r_{0}\right)=0$, i.e. the same as that for $m=0$ and $W_{0}=0$. Similarly, the fact that the growth rate goes to zero for the azimuthal wavenumber cut-off $m= \pm m_{c}$ when $W_{0}=0$ implies that a given azimuthal wavenumber $m$ for swirling jets will be unstable in the range of axial wavenumbers: $-\left(m_{c}+m\right) / W_{0}<k<\left(m_{c}-m\right) / W_{0}$. Since $k$ and $W_{0}$ are assumed positive by convention, this indicates that all of the azimuthal wavenumbers $m<m_{c}$ are expected to be unstable. These predictions will be checked numerically in $\S 6.2$. In contrast, we can remark that if the axial flow is constant $W(r)=W_{0}$, the stability properties (wavenumber selection and growth rate) will remain the same as without axial flow. The only modification is a Doppler shift of the frequency by $k W_{0}$.

In the general case of axial and angular velocity profiles with different shapes, we can determine the maximum growth rate and the most amplified axial wavenumber for a given azimuthal wavenumber $m$. To do so, we consider the derivative of the growth rate (4.21) with respect to $k$

$$
\frac{\partial \sigma}{\partial k}=-\mathrm{i} W\left(r_{0}\right)+O\left(\frac{1}{k^{2}}\right) .
$$

We see that the real part of the growth rate for a given $m$ will reach an extremum if $r_{0}$ is real. Equation (4.22) then implies that we must have simultaneously $\phi^{\prime}\left(r_{0}\right)=0$ and $k W^{\prime}\left(r_{0}\right)+m \Omega^{\prime}\left(r_{0}\right)=0$. If we assume that $\phi$ has a negative minimum at a real radius $r_{0 m}$, the first condition is satisfied if $r_{0}=r_{0 m}$ while the second one gives the corresponding axial wavenumber

$$
k=k_{m} \equiv-m \Omega^{\prime}\left(r_{0 m}\right) / W^{\prime}\left(r_{0 m}\right) .
$$

Since $k$ is positive, this condition can be satisfied only for negative azimuthal wavenumbers when $\Omega^{\prime}\left(r_{0 m}\right)$ and $W^{\prime}\left(r_{0 m}\right)$ are both negative. Furthermore, we have for $r_{0}=r_{0 m}$

$$
\frac{\partial^{2} \sigma}{\partial k^{2}}=\frac{2 W^{\prime 2} \phi}{\phi^{\prime \prime} \sqrt{-\phi}-\mathrm{i} m \Phi\left(\Omega^{\prime \prime} W^{\prime}-W^{\prime \prime} \Omega\right) / W^{\prime}} .
$$


This shows that $\operatorname{Re}\left(\partial^{2} \sigma / \partial k^{2}\right)<0$ since $\phi^{\prime \prime}\left(r_{0 m}\right)>0$. Thus, $k=k_{m}$ is a local maximum of the growth rate curve $\sigma_{r}(k, m)$ for a given azimuthal wavenumber $m$. The corresponding growth rate is $\sigma_{r \max }=\sqrt{-\phi\left(r_{0 m}\right)}$. These results mean that, as soon as a small axial flow is added to a centrifugally unstable vortex, the growth rate for a given negative azimuthal wavenumber will be maximum for a finite axial wavenumber $k_{m}$ in contrast to pure vortices.

Physically, the condition (4.24) means that the growth rate is maximum when the wavenumber vector $\boldsymbol{Q}$ of the perturbation is parallel to the direction of zero strain of the base flow (Emanuel 1984; Stewartson \& Leibovich 1987). When there is no axial flow, this condition can be satisfied only for $m=0$ whereas, as soon as the axial flow is non-zero, equation (4.24) can be fulfilled for any negative azimuthal wavenumber. The corresponding maximum growth rate is then independent of $m$ and the magnitude of the axial flow. A small axial flow has therefore only a Doppler shifting effect: it modifies the wavenumber selection without affecting the maximum growth rate. When the axial flow is larger, we shall see in $\S 6.2$ that its main effect remains the same but there is also an increase of the maximum growth rate.

\section{Maximum growth rate}

When $\Lambda^{\prime \prime}=O(1)$, Leibovich \& Stewartson (1983) have shown that the maximum growth rate is given by the maximum of $\sqrt{-\tilde{\Phi}}$ where $\tilde{\Phi}$ is defined in (4.5). As shown in the previous section, this result also holds when the axial flow is small such that $W=O(1 / \kappa)$ since $\tilde{\Phi} \sim \phi$ in this limit. The question is now to determine whether or not this result also applies to arbitrary velocity profiles and in particular to the axial velocity profile (4.7) with $\epsilon$ small. To answer this question, we seek the maximum of the growth rate for a given azimuthal wavenumber $m$ using the general formulae (3.25) and (3.22). Deriving (3.25) with respect to $k$ gives

$$
\frac{\partial \sigma}{\partial k}=-\mathrm{i} W\left(r_{0}\right)-\frac{\partial \Phi /\left.\partial k\right|_{r}\left(r_{0}\right)}{2 \sqrt{-\Phi\left(r_{0}\right)}}+\frac{\partial \delta \sigma}{\partial k} .
$$

The second term in the right-hand side of (5.1) is small because $\partial \Phi /\left.\partial k\right|_{r}=O(1 / k)$ and $\Phi\left(r_{0}\right)=O(1)$. Note that the axial wavenumber can be considered here to be large $k=O(\kappa)$ since it is assumed that $O(W)=O(\Omega)=1$. The last term is also small but its precise order of magnitude depends on $O\left(\Lambda^{\prime \prime}\right)$ : it is $O\left(1 / k^{3 / 2}\right)$ when $\Lambda^{\prime \prime}=O(1)$ whereas it is $O(1 / k)$ for the profile (4.7) when $\epsilon=O(1 / k)$. Since we assume that $W=O(1)$, we can deduce from (5.1) that the maximum growth rate will be reached when the imaginary part of $r_{0}$ is small: $r_{0}=r_{0 r}+\mathrm{i} r_{0 i}$ with $r_{0 i}=O(1 / k)$. It follows that the maximum growth rate is given by $\sigma_{r \max }=\sqrt{-\Phi\left(r_{0 r}\right)}+O(1 / k)$.

In order to determine $r_{0 r}$, we first compute $r_{0 i}$ from (5.1) by imposing $\partial \operatorname{Re}(\sigma) / \partial k=$ 0 :

$$
r_{0 i} \sim \frac{\partial \Phi /\left.\partial k\right|_{r}\left(r_{0 r}\right)}{2 W^{\prime}\left(r_{0 r}\right) \sqrt{-\Phi\left(r_{0 r}\right)}}-\frac{\operatorname{Re}(\partial \delta \sigma / \partial k)}{W^{\prime}\left(r_{0 r}\right)} .
$$

When the real part of $r_{0}$ is not too small so that $r_{0 r} \gg r_{0 i}$, the relation (3.22) can be expanded into

$$
\begin{gathered}
\Lambda^{\prime}\left(r_{0 r}\right)=0, \\
2 \Lambda^{\prime \prime}\left(r_{0 r}\right) r_{0 i}=\frac{\Phi^{\prime}\left(r_{0 r}\right)}{\kappa \sqrt{-\Phi\left(r_{0 r}\right)}},
\end{gathered}
$$


for the real and imaginary parts, respectively. As a matter of fact, these two relations correspond to the first and second orders of the expansion (4.1) of Leibovich \& Stewartson (1983).

By using (5.2) and (5.3), equation (5.4) can be rewritten

$$
\tilde{\Phi}^{\prime}\left(r_{0 r}\right)=-2 \kappa \operatorname{Re}\left(\frac{\partial \delta \sigma}{\partial k}\right) \frac{\Lambda^{\prime \prime}\left(r_{0 r}\right)}{W^{\prime}\left(r_{0 r}\right)} \sqrt{-\Phi\left(r_{0 r}\right)},
$$

where $\tilde{\Phi}$ is defined in (4.5). When $\Lambda^{\prime \prime}\left(r_{0 r}\right)=O(1)$, the right-hand side of (5.5) is $O\left(k^{-1 / 2}\right)$ because $\partial \delta \sigma / \partial k=O\left(k^{-3 / 2}\right)$. Therefore, we retrieve the fact that maximum growth is achieved for large wavenumbers for the real radius $r_{0 r}$ for which $\tilde{\Phi}^{\prime}=0$ as found by Leibovich \& Stewartson (1983) (see $\S 4.1$ ). In the case of the profile (4.7) with $\epsilon=O(1 / k)$, the term $\partial \delta \sigma / \partial k$ is higher: $O(1 / k)$ but we have also $\Lambda^{\prime \prime}\left(r_{0 r}\right)=O(1 / k)$ in this case. It follows that the right-hand side of (5.5) is also negligible for large wavenumber. Therefore, the maximum growth rate is always $\sigma_{r \max }=\sqrt{-\min (\tilde{\Phi}(r))}$ with $r$ real regardless of the velocity profiles.

Nevertheless, it should be emphasized that (5.5) relies upon the hypothesis that $r_{0 r} \gg r_{0 i}$, i.e. $r_{0 r} \gg 1 / k$. When $r_{0 r}$ becomes small such that $r_{0 r}=O(1 / k)$, the relation (5.2), the expansion (5.3)-(5.4) and the resulting relation (5.5) cease to be valid. In addition, we see from (5.2) that $r_{0 i}$ can become large when $\Phi$ is small. Hence, when either $r_{0}$ or $\Phi$ are small, the maximum growth rate may not correspond to the minimum of $\tilde{\Phi}$ and an instability may even exist if $\tilde{\Phi}>0$. This happens for the Batchelor vortex when $q$ is slightly beyond $\sqrt{2}$. In this range, $\tilde{\Phi}$ is always positive (Leibovich \& Stewartson 1983) but numerical stability analyses show that there is still an instability for finite $m$. Interestingly, we shall see in $\S 6.1$ that (3.25) continues to predict a positive growth rate for finite $m$ slightly beyond $q=\sqrt{2}$. However, since the radius $r_{0}$ and $\Phi$ are then small, equation (3.25) is also not rigourously valid. In fact, a specific asymptotic analysis should be carried out directly from (2.2) with the assumptions $r \ll 1$ and $\Phi \ll 1$ as done by Stewartson \& Leibovich (1987).

\section{Comparison with numerical results}

The asymptotic predictions have been checked for two types of vortex profiles by solving numerically (2.2) using a shooting method. Following Leibovich \& Stewartson (1983), the path of integration is deformed in the complex plane to avoid the singular points where $S=0$ with the contour

$$
r=x\left[1-\mathrm{i} \delta\left(1-\frac{x}{x_{2}}\right)\left(k W^{\prime}(x)+m \Omega^{\prime}(x)\right)\right],
$$

where $x$ is a real variable, $\delta$ a control parameter and $x_{2}$ the outer integration limit. This allows to compute near neutral modes for which the singularities are close to the real $r$-axis (Leibovich \& Stewartson 1983; Mayer \& Powell 1992). Note that $\delta$ should be positive in order to avoid the critical points in the correct direction in the complex $r$-plane. Equation (2.2) is integrated from a small value $x_{1}$ and from $x_{2}$ toward a fitting point $x_{f}$ using an initial guess for $\sigma$. The integrations are started at $x_{1}$ and $x_{2}$ by using the asymptotic solutions of (2.2) satisfying the boundary conditions: $u_{r} \sim r^{|| m|-1|}$ as $r \rightarrow 0$ and $u_{r} \sim K_{|m|}^{\prime}(k r)$ as $r \rightarrow \infty$. An iterative Newton method is then used to find the value of $\sigma$ for which the Wronskian at $x_{f}$ vanishes. The numerical results have been checked against an independent Chebyshev pseudo-spectral collocation method. 

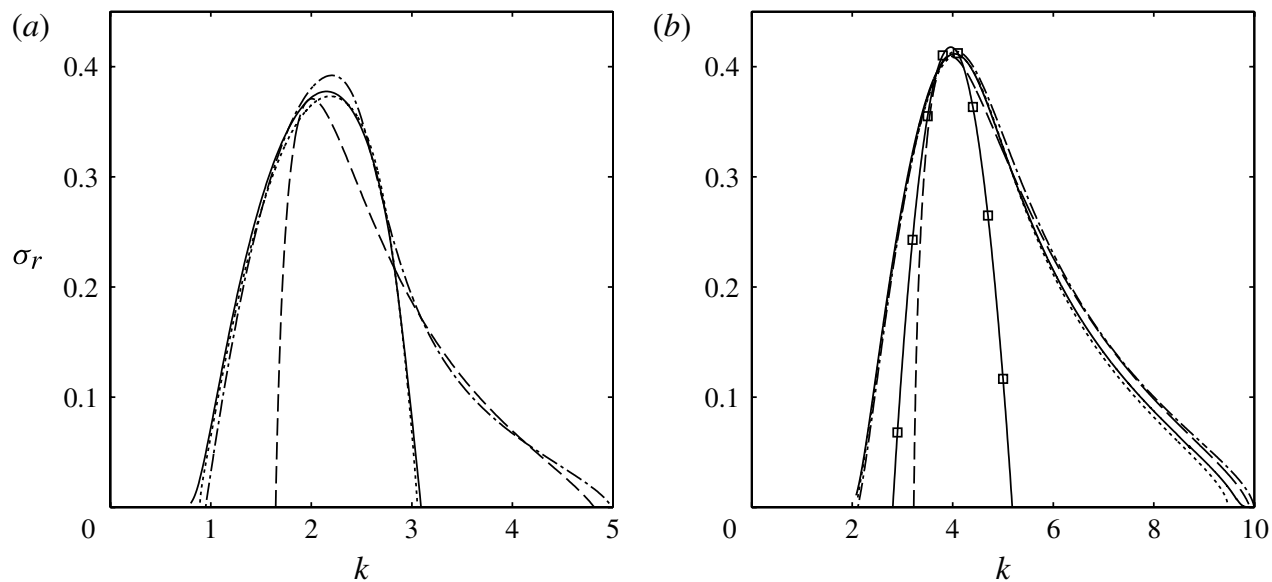

FIGURE 1. Growth rate $\sigma_{r}$ for the azimuthal wavenumbers $(a) m=-4$ and $(b) m=-8$ as a function of the axial wavenumber $k$ for the Batchelor vortex for $q=0.8$. The exact growth rate is shown by a solid line and the different asymptotic approximations are shown: - - , (3.25); ---, Leibovich and Stewartson formula (D 1); $\cdots,(6.2)$. In (b), the asymptotic formula (4.20) of Di Pierro \& Abid (2010) is also plotted with a solid line marked with square symbols.

Concerning the asymptotics, equations (3.22) or (4.2) governing the location of the stationary point $r_{0}$ are solved by an iterative Newton method. Since there may exist several unphysical stationary points in the complex plane, a convenient method to obtain a correct initial guess value for the Newton method is to first compute $r_{0}$ for an axial wavenumber close to the most amplified axial wavenumber. As shown in $\S 5$, the stationary point $r_{0}$ is then close to the real radius $r_{0 r}$ where $\tilde{\Phi}$ is minimum. Hence, the radius $r_{0 r}$ is first searched and the corresponding axial wavenumber is computed from $k_{0}=-m \Omega^{\prime}\left(r_{0 r}\right) / W^{\prime}\left(r_{0 r}\right)$. The radius $r_{0 r}$ is then used as guess value of the Newton method for $k=k_{0}$. Subsequently, the axial wavenumber is varied continuously by small steps from $k_{0}$ and, at each step, the value of $r_{0}$ found for the previous value of $k$ is used as a guess value.

We first present the results for the Batchelor vortex since its stability properties in the inviscid limit have been studied extensively numerically (Lessen et al. 1974; Mayer \& Powell 1992). The Carton \& McWilliams (1989) vortex profiles with an axial flow will be presented next. In contrast to the Batchelor vortex, such vortex is centrifugally unstable even when there is no axial flow so that we shall be able to study the effect of a small axial flow on the centrifugal instability as described theoretically in $\S 4.4$.

\subsection{Batchelor vortex}

We first remind that, for the Batchelor vortex (1.3), there is a band of unstable axial wavenumbers $k$ for each negative azimuthal wavenumber provided that $q \lesssim 1.5$ (Lessen et al. 1974; Mayer \& Powell 1992). The most amplified axial wavenumber $k_{\max }$ increases with $|m|$. For moderate azimuthal wavenumbers, there also exist unstable centre modes up to $q=2.31$ but their growth rate is very small (Stewartson \& Brown 1985; Heaton 2007a).

As an example, figures $1(a)$ and 2(a) show the maximum growth rate and the corresponding frequency obtained numerically (solid line) and by the various asymptotic formulae (other lines) for $q=0.8$ and $m=-4$. We see that all of 
(a)

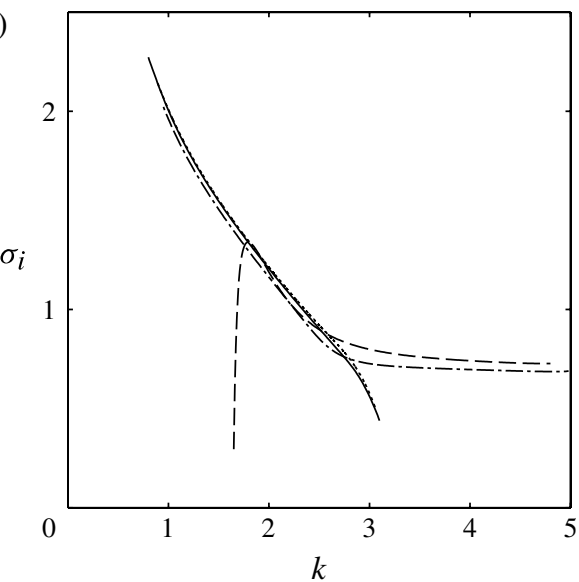

(b)

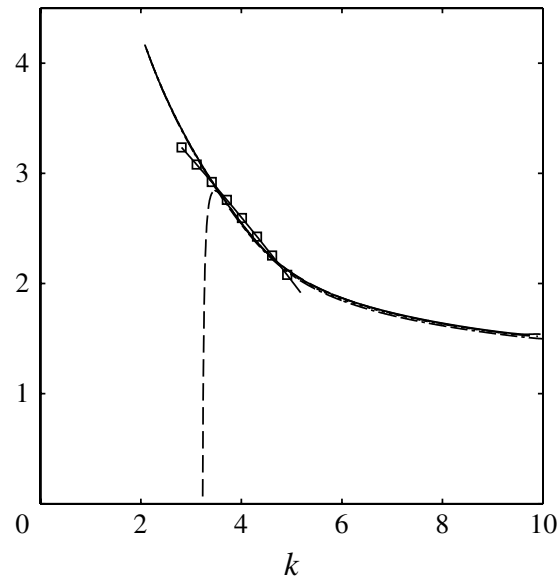

FIGURE 2. Frequency $\sigma_{i}$ for the azimuthal wavenumbers $(a) m=-4$ and $(b) m=-8$ as a function of the axial wavenumber $k$ for the Batchelor vortex for $q=0.8$. The exact frequency is shown by a solid line and the different asymptotic approximations are shown: $-\cdot-,(3.25)$; --- , Leibovich and Stewartson formula (D 1); $\cdots,(6.2)$. In (b), the asymptotic formula (4.20) of Di Pierro \& Abid (2010) is also plotted with a solid line marked with square symbols.

the asymptotic results are generally in quite good agreement with the numerical results although the azimuthal and axial wavenumbers are of order unity, i.e. not large as assumed in the asymptotic analyses. However, some differences can be noticed between the various asymptotic predictions. In particular, the growth rate (D 1) of Leibovich \& Stewartson (1983) (dashed line) departs from the exact growth rate for low axial wavenumbers (figure $1 a$ ) and goes to zero at $k=-m q / 2=1.6$. The associated frequency also vanishes and differs widely from the exact frequency (figure 2a). In contrast, the formula (3.25) (dash-dotted line), despite its lower formal accuracy in wavenumber, provides a very good approximation of the growth rate and frequency down to the lower wavenumber cut-off $k_{c} \approx 1$. The departure of the Leibovich \& Stewartson (1983) growth rate comes from the fact that the radius $r_{00}$ given by (4.2) becomes small and vanishes for $k=-m q / 2$. In fact, we are in the situation where $\Lambda^{\prime \prime}\left(r_{0}\right)$ is small and the estimation of $r_{0}$ by (4.2) is inaccurate. In contrast, the radius given by (3.22) is not small for finite wavenumbers: for example, it is $r_{0}=0.6162-0.2731 \mathrm{i}$ for $m=-4$ and $k=1.3$. Stewartson \& Leibovich (1987) have performed a specific asymptotic analysis for this limiting case but we see that (3.25) provides a good prediction without any additional difficulty.

The growth rate peak is slightly overestimated by (3.25) and underestimated by (D 1). Its location is well predicted by (3.25) and is slightly shifted to the left by (D 1).

For high axial wavenumbers (figure $1 a$ ), the asymptotic growth rates (D 1) and (3.25) both differ from the exact growth rate and go to zero near $k=-m / q \approx 5$ while the exact growth rate vanishes at $k \approx 3$. This is because the generalized Rayleigh discriminant $\Phi\left(r_{0}\right)$ becomes small there and vanishes for $k=-m / q$. Therefore, the assumption that $S\left(r_{0}\right)=O(1)$ ceases to be valid. Alternatively, the use of the latter hypothesis can be easily avoided by solving directly the implicit relations (3.7) and (3.13) at leading orders, i.e.

$$
\frac{\partial B_{0}}{\partial r}\left(r^{*}\right)+\frac{1}{\kappa} \frac{\partial B_{1}}{\partial r}\left(r^{*}\right)=0
$$



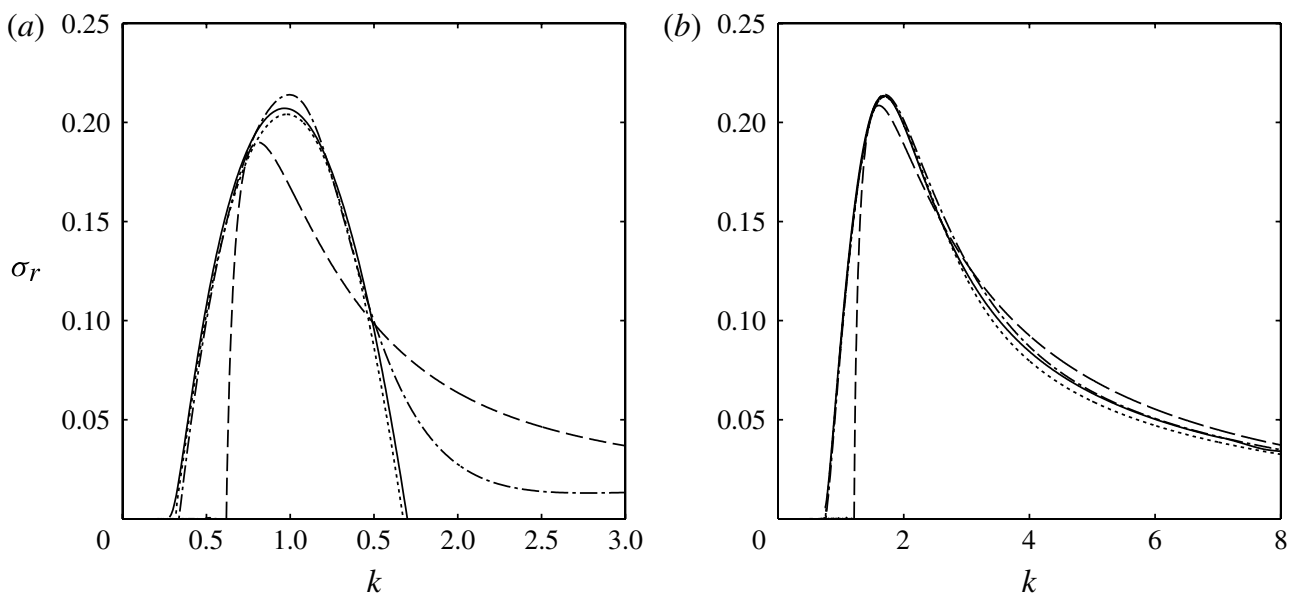

FIGURE 3. Growth rate $\sigma_{r}$ for the azimuthal wavenumbers $(a) m=-4$ and $(b) m=-8$ as a function of the axial wavenumber $k$ for the Batchelor vortex for $q=0.3$. The exact growth rate is shown by a solid line and the different asymptotic approximations are shown: - - , (3.25); ---, Leibovich \& Stewartson formula (D 1); ‥, (6.2).

$$
B_{0}\left(r^{*}\right)+\frac{B_{1}\left(r^{*}\right)}{\kappa}=-\frac{2 n+1}{\sqrt{2} \kappa} \sqrt{\frac{\partial^{2} B_{0}}{\partial r^{2}}\left(r^{*}\right)+\frac{1}{\kappa} \frac{\partial^{2} B_{1}}{\partial r^{2}}\left(r^{*}\right)} .
$$

The corresponding growth rate and frequency are shown by a dotted line in figures 1(a) and 2(a). We see that the agreement with the exact growth rate is excellent over the whole unstable band. Note that an explicit asymptotic formula for the growth rate when $\Phi$ is small is also derived and tested in appendix C.

Figures $1(b)$ and $2(b)$ show the growth rate and frequency for a higher azimuthal wavenumber: $m=-8$ for the same swirl parameter $q=0.8$. As expected, the discrepancies between the numerical and asymptotic results are reduced since the wavenumbers are doubled. Nevertheless, the departure of the growth rate (D 1) of Leibovich \& Stewartson (1983) is still visible for low axial wavenumbers. For high axial wavenumbers, equations (D 1) and (3.25) are now both in good agreement with the exact growth rate. These two approximations are close because $\Lambda^{\prime \prime}\left(r_{0}\right)=O(1)$ in this region so that the estimate of $r_{0}$ by (4.2) is accurate. Again, the growth rate predicted by the implicit dispersion relation (6.2) is in remarkable agreement with the exact growth rate.

Even if the assumption (4.15) of slowly varying angular and axial velocity profiles does not apply to the Batchelor vortex, the asymptotic formula (4.20) of Di Pierro \& Abid (2010) is also shown by a solid line marked with square symbols in figures $1(b)$ and $2(b)$. The growth rate (4.20) agrees with the exact growth rate and the other asymptotic formulae only close to the maximum. This is because the assumption (4.15) used to obtain (4.20) implies $\Phi^{\prime}\left(r_{0}\right) \simeq 0$ (see (4.18)), a relation which is also fulfilled by the asymptotic formulae (3.25) and (D 1) near the growth rate peak as shown in $\S 5$. Away from the peak, the relation $\Phi^{\prime}\left(r_{0}\right) \simeq 0$ is no longer valid for the Batchelor vortex explaining why (4.20) departs from (D 1) or (3.25).

Similar comparisons for other values of the swirl parameter $q$ are shown in figures 3 and $4(q=0.3)$, figure $5(q=1.3)$ and figure $6(q=1.4)$. In all of these examples, the growth rate (D 1) (Leibovich \& Stewartson 1983) goes to zero at $k=-m q / 2$ and 

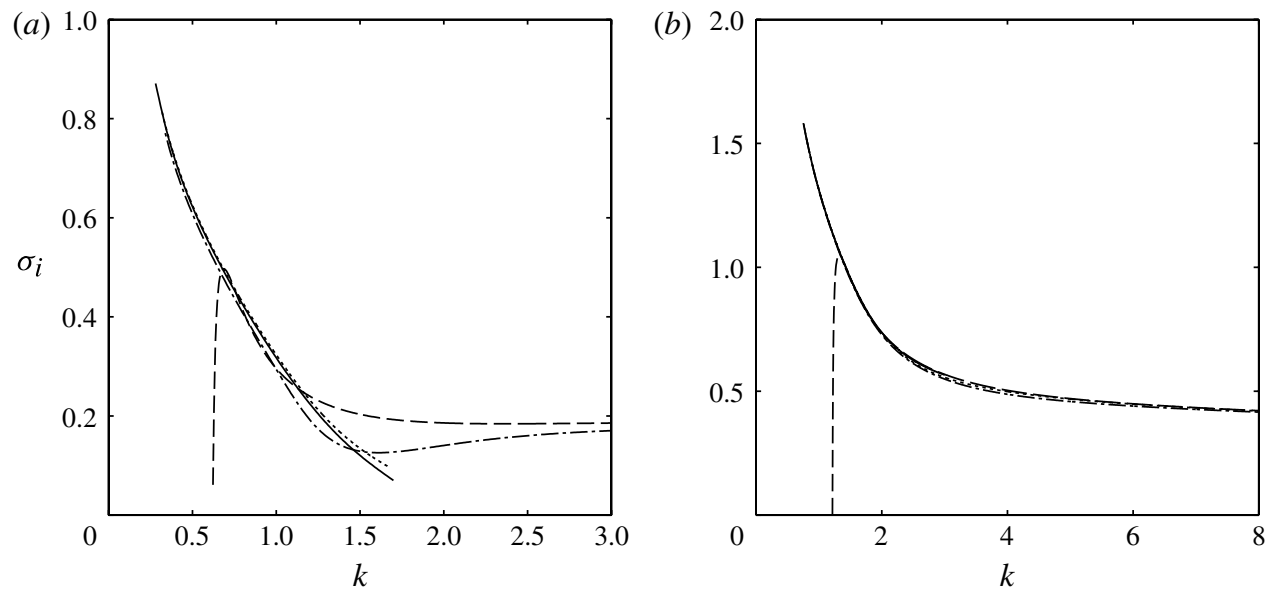

FIGURE 4. Frequency $\sigma_{i}$ for the azimuthal wavenumbers $(a) m=-4$ and $(b) m=-8$ as a function of the axial wavenumber $k$ for the Batchelor vortex for $q=0.3$. The exact frequency is shown by a solid line and the different asymptotic approximations are shown: $-\cdot-,(3.25)$; --- , Leibovich and Stewartson formula (D 1); $\cdots,(6.2)$.
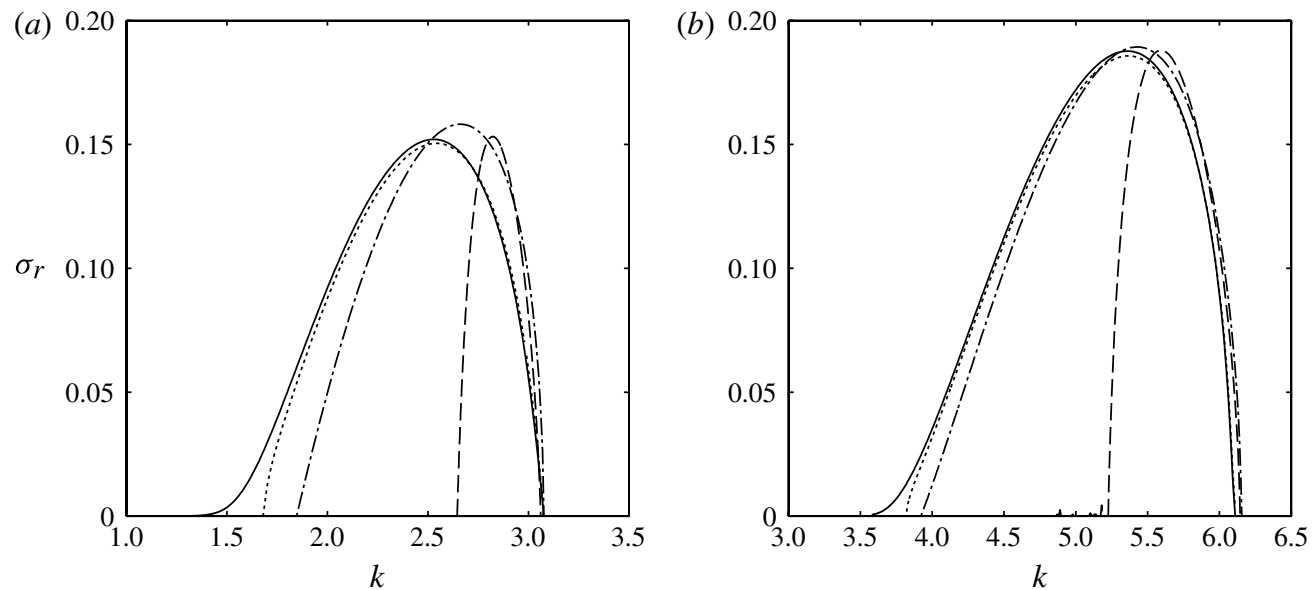

FIGURE 5. Similar to figure 3 but for $q=1.3$.

fails to describe the exact growth rate for low axial wavenumbers. This breakdown is particularly visible for the frequency (only shown for $q=0.3$, figure 4). For $q=0.3$ and $m=-4$ (figure 3), the growth rate (D 1) and (3.25) decrease slowly as $k$ increases while the exact growth rate goes quickly to zero at $k \approx 1.7$. This discrepancy disappears when solving directly the implicit dispersion relation (6.2).

For $q=1.3$ and $q=1.4$, the results obtained from (6.2) are again in excellent agreement with the numerical results except near the lower neutral point. The formula (3.25) also provides quite a good approximation for $q=1.3$ (figure 5) but deteriorates for $q=1.4$ (figure 6) all the more when $m$ is low. This is because $\Phi$ is small over the whole instability curve. The growth rate (D 1) of Leibovich \& Stewartson (1983) is also correct near the upper cut-off $k=-m / q$. However, the lower cutoff $k=-m q / 2$ 
(a)

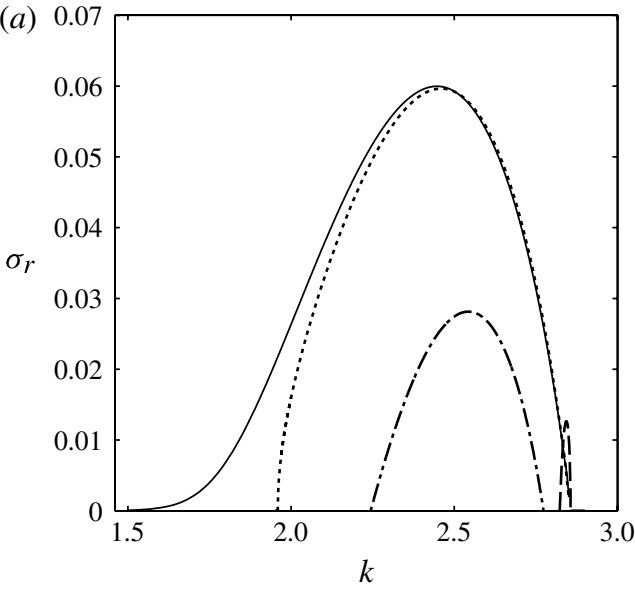

(b)

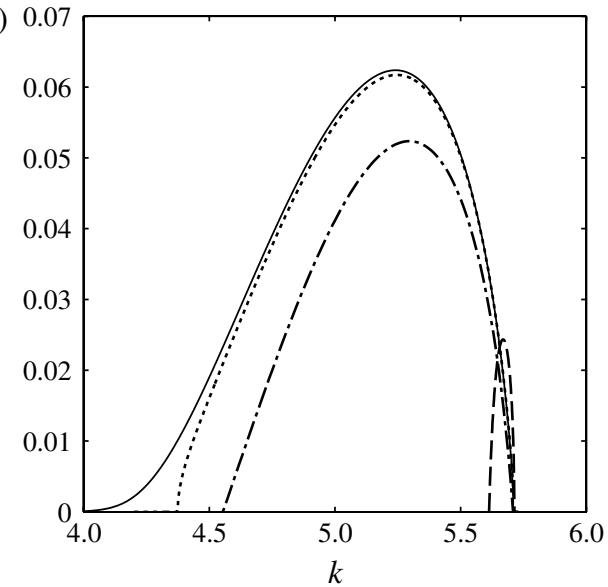

FIGURE 6. Similar to figure 3 but for $q=1.4$.

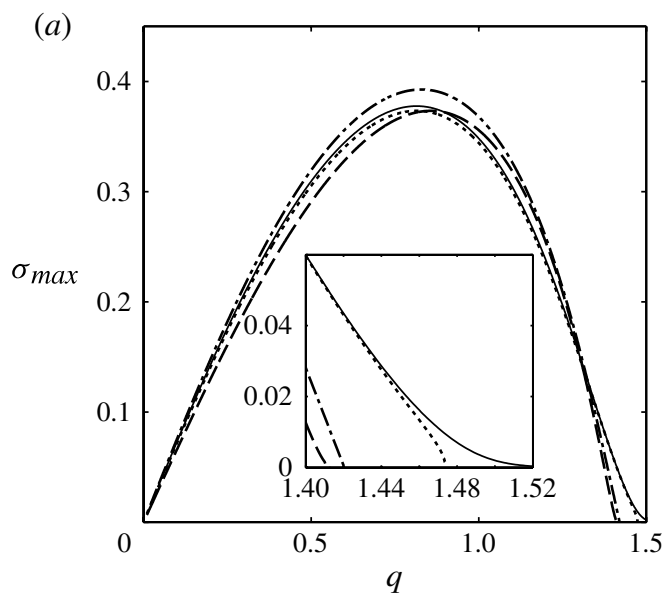

(b)

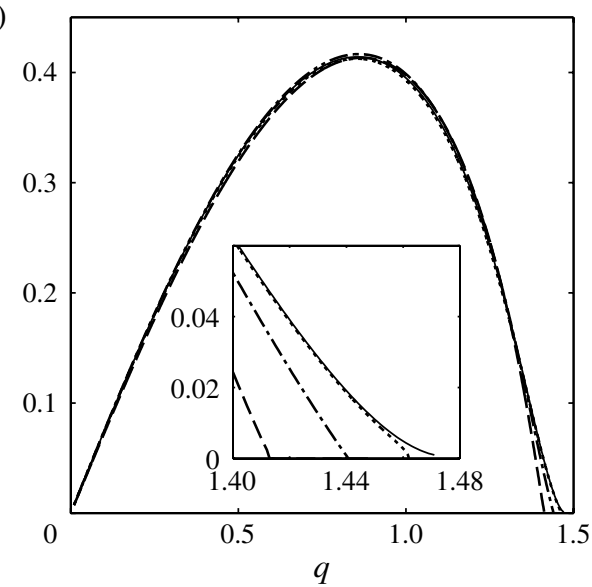

FIGURE 7. Maximum growth rate $\sigma_{r \max }$ for $(a) m=-4$ and $(b) m=-8$ as a function of $q$. The exact growth rate is shown by a solid line and the different asymptotic approximations are shown by: $-\cdot-,(3.25) ;---$, Leibovich and Stewartson formula (4.4); $\cdots,(6.2)$. The inset shows a close-up view of the range $q=1.4-1.5$.

predicted by Leibovich \& Stewartson (1983) is clearly not valid and the growth rate (D 1) becomes less and less correct as $q$ increases even for $m=-8$ (figures $5 b$ and $6 b$ ).

Leibovich \& Stewartson (1983) have deduced their stability threshold $q=\sqrt{2}$ for the Batchelor vortex from the band of unstable axial wavenumbers predicted by (D 1): $-m q / 2<k<-m / q$. However, as already mentioned, the actual critical value is slightly larger $q \approx 1.5$ (we exclude the centre modes that are of different nature). The origin of this discrepancy can be simply understood from figures 5 and 6 since we see that the lower axial wavenumber cutoff is actually smaller than $-m q / 2$.

In order to further investigate this issue, we show in figure 7 the maximum growth rate over $k$ as a function of the swirling parameter $q$ for $m=-4$ and $m=-8$. 
For $m=-4$, the maximum growth rate predicted by (3.25) and (D 1) vanish around the same value of $q: q=1.42$ and $q=\sqrt{2}$, respectively. However, it should be emphasized that these two approximations are actually very different around $q=1.4$ as seen in figure 6(a). The implicit dispersion relation (6.2) is in remarkable agreement with the numerical result up to $q=1.46$. For $m=-8$ (figure $7 b$ ), the critical swirl parameter predicted by (3.25) is higher $q \approx 1.44$ whereas the Leibovich \& Stewartson (1983) growth rate still goes to zero at $q=\sqrt{2}$ since this threshold is independent of $m$. Again, (6.2) is in remarkable agreement with the exact result up to $q \approx 1.46$.

In summary, these examples show that the asymptotic growth rate (3.25) is more accurate than the one obtained previously by Leibovich \& Stewartson (1983) even if its formal accuracy in terms of the wavenumber is lower. The breakdown of the Leibovich \& Stewartson (1983) growth rate is mostly due to the expansion (4.1) which turns out to be inaccurate when the shapes of the angular and axial velocity profiles are close in the neighbourhood of the stationary point $r_{0}$. When $\Phi$ is small, the two asymptotic predictions (3.25) and (D 1) are not very good but the implicit relation (6.2) yields excellent results.

\subsection{Carton and McWilliams vortices with axial flow}

We now consider the Carton \& McWilliams (1989) family of vortices with an axial flow superimposed:

$$
\begin{gathered}
U_{\theta}(r)=r e^{-r^{\alpha}}, \\
W(r)=W_{0} e^{-r^{\gamma}},
\end{gathered}
$$

where $\alpha, \gamma$ and $W_{0}$ are constants.

When there is no axial flow $W_{0}=0$, such vortex is known to be unstable to the centrifugal instability as soon as $\alpha>0$ (Smyth \& McWilliams 1998; Gallaire \& Chomaz 2003; Billant \& Gallaire 2005). The latter authors have shown that the band of unstable azimuthal wavenumbers is $0 \leqslant|m|<2 \sqrt{\alpha}$. This can be seen in figure 8 where the growth rates for $\alpha=2$ with $W_{0}=0$ are plotted as a function of $k$ with dotted lines. Only the azimuthal wavenumbers $m=0,|m|=1$ and $|m|=2$ are unstable for $\alpha=2$. The maximum growth rate for a given azimuthal wavenumber is reached for $k \rightarrow \infty$ and the dominant azimuthal wavenumber is the axisymmetric mode $m=0$.

When a small axial flow $W_{0}=0.05$ is applied with $\gamma=2$, the stability properties (solid lines in figure 8) look completely different. The growth rate of a given azimuthal wavenumber is maximum for a finite axial wavenumber and the symmetry between positive and negative azimuthal wavenumbers is broken. The positive azimuthal wavenumbers $m=0, m=1$ and $m=2$ tend to be stabilized whereas all of the negative azimuthal wavenumbers are destabilized. The maximum growth rate of the negative azimuthal wavenumbers lie on the growth rate curve of the $m=0$ mode for $W_{0}=0$. The corresponding most amplified axial wavenumber increases with $|m|$.

As shown by the dash-dotted lines, the WKB prediction (3.25) (the formula (4.21) is not shown since it gives almost the same result) for $W_{0}=0.05$ is in very good agreement with the numerical results when $k \gtrsim 5$. The discrepancies at low axial wavenumbers are due to the large wavenumber hypothesis of the asymptotic analysis and also to the fact that the $|m|=2$ wavenumbers are unstable at $k=0$ because of the shear instability. Therefore, the theoretical results obtained in $\S 4.4$ are fully confirmed. The growth rate of negative azimuthal wavenumbers is maximum for the axial wavenumber given by (4.24) and is equal to $\sigma_{r \max }=\sqrt{-\phi\left(r_{0 m}\right)}+O(1 / k)$ for all of the negative azimuthal wavenumbers. 

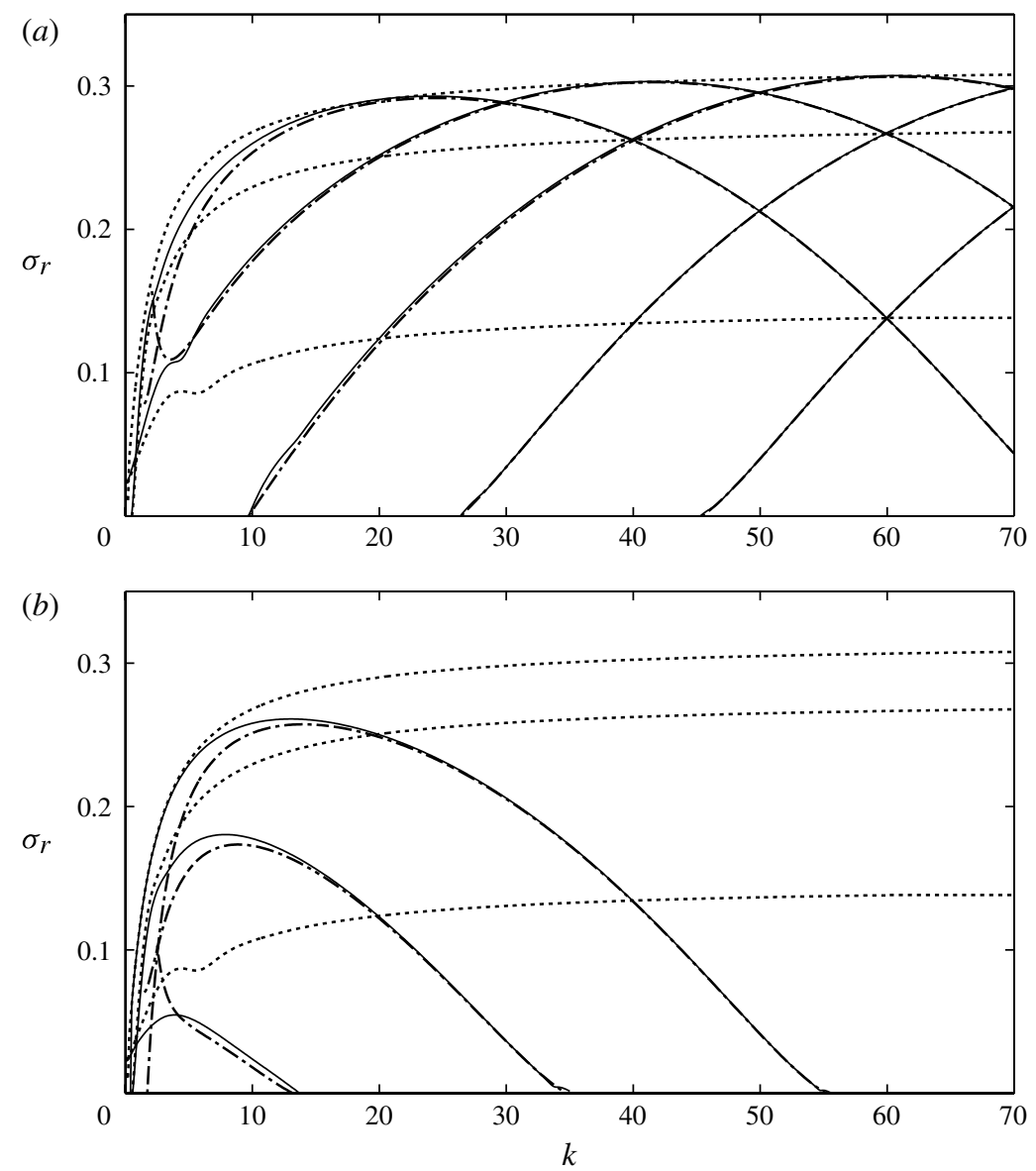

FIGURE 8. Growth rate $\sigma_{r}$ for (a) $m=-1,-2,-3,-4,-5$ (from left to right) and (b) $m=0,1,2$ (from top to bottom) as a function of the axial wavenumber $k$ for the basic flow (6.3) with $\alpha=2, \gamma=2$ and for $W_{0}=0$ (dotted lines) and $W_{0}=0.05$ (solid lines). The asymptotic approximation (3.25) for $W_{0}=0.05$ is plotted with dash-dotted lines.

The values $\alpha=2$ and $\gamma=2$ investigated above are peculiar since the angular and axial velocity profiles (6.3) have exactly the same shape. As shown in $\S 4.4$, the effect of a small axial flow is somewhat trivial in this case because the growth rate can be deduced from that for $W_{0}=0$ by applying the transformation $m \rightarrow m+k W_{0}$. Moreover, the asymptotic formula of Leibovich \& Stewartson (1983) completely breaks down as discussed in $\S 3$ since a single axial wavenumber $k=-m / W_{0}$ satisfies (4.2). To some extent, this can be interpreted as the limit for $m \rightarrow-\infty$ of (3.25) and (3.22). Indeed, the unstable band is centred on $k / m=-1 / W_{0}$ but its relative width $-m_{c} /\left(m W_{0}\right)-1 / W_{0}<k / m<-m_{c} /\left(m W_{0}\right)-1 / W_{0}$ shrinks to zero as $m \rightarrow-\infty$.

A further comparison for $\alpha=4, \gamma=2$ and $W_{0}=0.3$, i.e. for different angular and axial velocity profiles, is shown in figure 9 . The agreement is good and improves as $m$ decreases. In contrast to the previous case, we can note that the maximum growth rate is slightly above the growth rate of the axisymmetric mode for $W_{0}=0$. This is because the term involving the axial velocity in $\Phi$ (see (3.3)) is no longer negligible since $W_{0}=0.3$. The growth rate (D1) of Leibovich \& Stewartson (1983) is also 

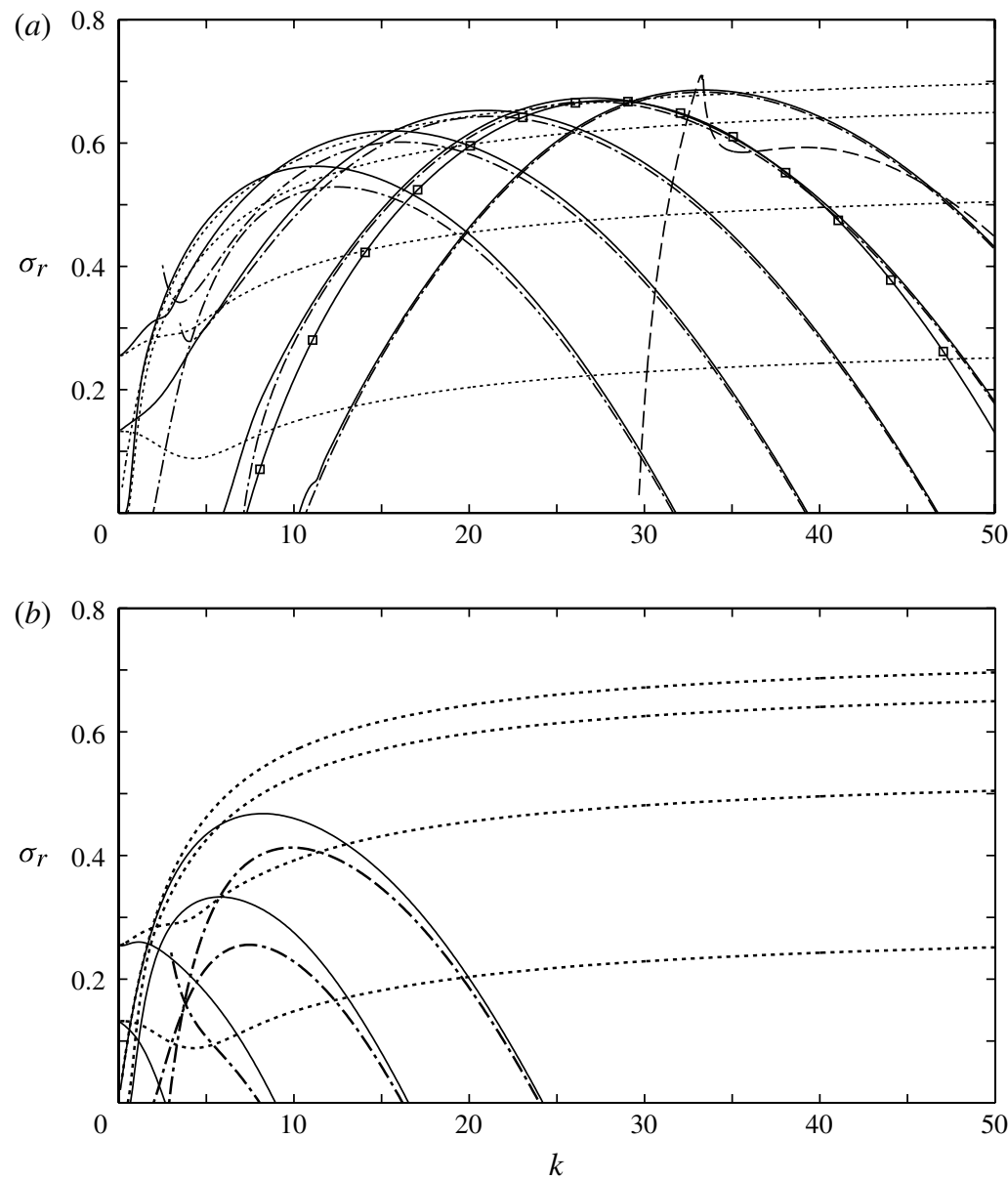

FIGURE 9. Growth rate $\sigma_{r}$ for $(a) m=-1,-2,-3,-4,-5$ (from left to right) and (b) $m=0,1,2,3$ (from top to bottom) as a function of the axial wavenumber $k$ for the basic flow (6.3) with $\alpha=4$ and $\gamma=2$ for $W_{0}=0.3$ (solid lines) and $W_{0}=0$ (dotted lines). The asymptotic approximation (3.25) is plotted with dash-dotted lines. The asymptotical growth rate (D 1) of Leibovich \& Stewartson (1983) is shown by a dashed line for $m=-5$ only. The asymptotic formula (4.20) of Di Pierro \& Abid (2010) is also plotted with a solid line marked with square symbols for $m=-4$ only.

plotted for $m=-5$. As can be seen, it departs largely from the exact growth rate. The formula (D 1) diverges near $k=-m \alpha /\left(\gamma W_{0}\right)=33.3$ because $\Lambda^{\prime}\left(r_{0}\right)=\Lambda^{\prime \prime}\left(r_{0}\right)=0$ for this axial wavenumber. In addition, we can remark that the growth rate (D 1) crosses the growth rate (3.25) very close to its maximum. As shown in $\S 5$, this comes from the fact that $r_{0}$ is almost real and close to the radius given by (4.2) when the growth rate (3.25) is maximum.

The asymptotic formula (4.20) of Di Pierro \& Abid (2010) is also shown for $m=-4$ by a solid line marked with square symbols in figure 9 . Like for the Batchelor vortex, the agreement is best near the growth rate maximum. Away from the peak, (4.20) departs from the exact growth rate but much less than for the Batchelor vortex (figure 1). This indicates that the approximation $\Phi^{\prime}\left(r_{0}\right) \simeq 0$, or equivalently $\Lambda^{\prime}\left(r_{0}\right) \ll 1$, holds better for the family of swirling jet profiles (6.3). 

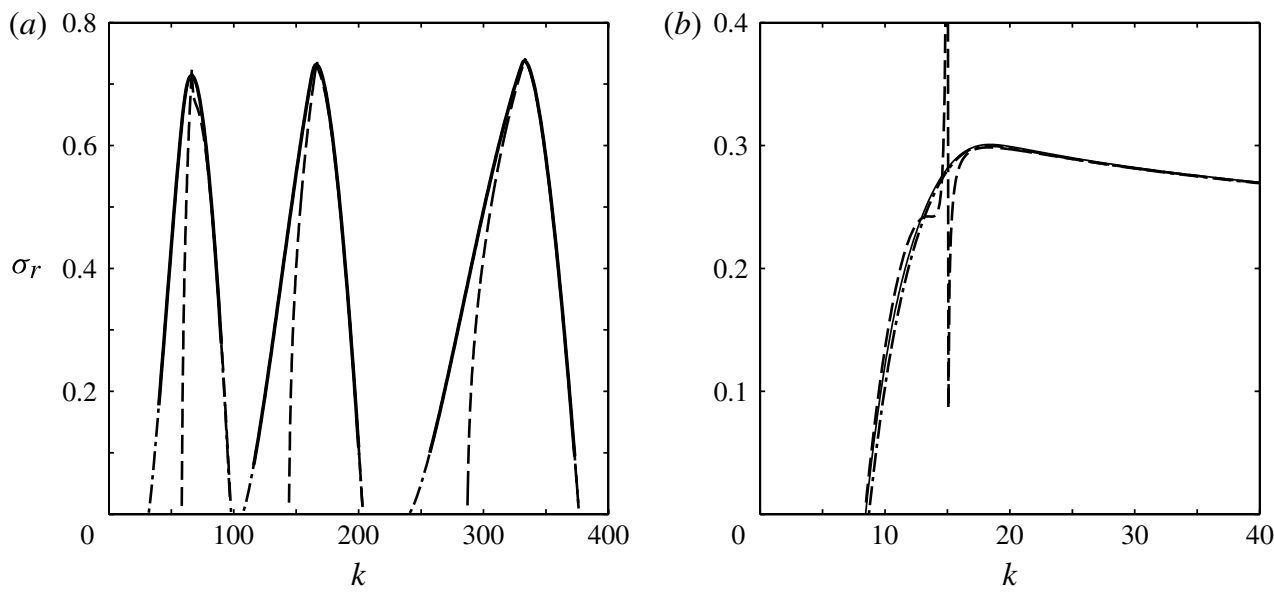

FIGURE 10. Growth rate $\sigma_{r}$ for the basic flow (6.3) as a function of the axial wavenumber $k$ for $(a) m=-10,-25,-50$ (from left to right) and $\alpha=4, \gamma=2, W_{0}=0.3$ and for (b) $m=-6$ and $\alpha=2, \gamma=4, W_{0}=0.2$. The exact growth rate is shown by solid lines. The asymptotic growth rate (3.25) and (D 1) are plotted with dash-dotted lines and dashed lines, respectively.

For higher azimuthal wavenumbers, the growth rate (D 1) of Leibovich \& Stewartson (1983) tends to the exact growth rate but the convergence as $m$ increases is very slow. Figure $10(a)$ shows a comparison for $m=-10, m=-25$ and $m=-50$ for the same parameters as before. Even for the latter azimuthal wavenumber, the growth rate (D 1) still differs from the exact growth rate in contrast to (3.25). In fact, it is only when $|m| \gtrsim 500$ than the formulae (D 1) and (3.25) become very close over the whole growth rate curve.

However, the convergence of the growth rate (D1) of Leibovich \& Stewartson (1983) as $m$ increases depends on each particular velocity profile. Figure 10(b) displays another comparison for $\alpha=2, W_{0}=0.2$ and $\gamma=4$ for a moderate azimuthal wavenumber $m=-6$. The breakdown of (D 1) when $\Lambda^{\prime \prime}\left(r_{0}\right)=0$ is much more localized and the agreement with the exact growth rate and the approximation (3.25) is good over a wide range of axial wavenumbers.

\section{Conclusion}

In summary, we have first shown that the WKB approach for the ring modes associated with the centrifugal instability of swirling jets leads to the same condition for large dimensionless wavenumber $\kappa=\sqrt{k^{2}+m^{2}}$ regardless of the magnitude of the axial flow:

$$
\kappa B\left(r^{*}\right)=-(2 n+1) \sqrt{\frac{B^{\prime \prime}\left(r^{*}\right)}{2}} \text { with } B^{\prime}\left(r^{*}\right)=0,
$$

where the function $B$ is defined in (3.1).

The main difference between swirling jets and pure vortices (i.e. vortices with no axial flow) concerns the Doppler shift function $\kappa \Lambda=m \Omega(r)+k W(r)$ contained in this WKB condition through the function $B$. The function $\kappa \Lambda$ is of order unity for pure vortices while it is large when there is an axial flow. However, an explicit asymptotic formula for the growth rate can be obtained from the WKB condition for arbitrary 
magnitude of $\kappa \Lambda$. This new formula (see (3.25) and (3.22)) is therefore uniformly valid whatever the magnitude of the axial flow and unifies the formulae of Leibovich \& Stewartson (1983) and Billant \& Gallaire (2005) for swirling jets and pure vortices, respectively.

When the axial flow is of order unity and the second derivative of the Doppler shift function $\kappa \Lambda^{\prime \prime}\left(r^{*}\right)$ is large, the growth rate formula simplifies (see (4.4) and (4.2)) and retrieves the leading orders of the asymptotic formula of Leibovich \& Stewartson (1983).

When the magnitude of the axial flow is small, the Doppler shift function is not large and the growth rate becomes similar to the formula of Billant \& Gallaire (2005) for pure vortices except that there is the additional term $k W(r)$ in $\kappa \Lambda$ (see (4.21) and (4.22)). This shows that a vortex which is centrifugally unstable remains unstable in the presence of an axial flow. However, even if the axial flow is small, it has a Doppler shifting effect which completely modifies the azimuthal wavenumber selection. All of the perturbations with negative azimuthal wavenumbers are destabilized whereas those with positive azimuthal wavenumbers tend to be stabilized (with the convention that the axial and angular flows $W, \Omega$ and the axial wavenumber $k$ are positive). The maximum growth rate for an azimuthal wavenumber $m$ is reached for the axial wavenumber $k=-m \Omega^{\prime}\left(r_{0}\right) / W^{\prime}\left(r_{0}\right)$ where $r_{0}$ is the radius where the Rayleigh discriminant $\phi$ is minimum. This corresponds to a wavenumber vector parallel to the direction of zero strain of the base flow.

We have also shown that the accuracy of the asymptotic formula of Leibovich \& Stewartson (1983) is strongly reduced when $\kappa \Lambda^{\prime \prime}\left(r^{*}\right)$ is not large. This happens whatever the magnitude of the axial flow as soon as the axial and angular velocity profiles have a similar shape. In contrast, the present growth rate formula (3.25) continues to provide accurate predictions. This is the reason why our asymptotical formula is accurate over a wider range of axial wavenumbers than the Leibovich-Stewartson asymptotics for the Batchelor vortex even if its formal accuracy in wavenumber is lower.

When the axial and angular velocity profiles are assumed to be constant or slowly varying, the formula (3.25) also recovers the asymptotic results of Leblanc \& Le Duc (2005) and Di Pierro \& Abid (2010) in the limit of an homogeneous and incompressible fluid. In the future, it would be interesting to extend the present analysis to variable density swirling compressible flows.

\section{Acknowledgements}

We thank S. Le Dizès and the anonymous referees for helping to improve the manuscript.

\section{Appendix A. Limits of a slowly rotating jet and a vortex with a small axial flow}

In this appendix, we study the order of magnitude of the function $B$ in the particular limits of a slowly rotating jet $[O(\Omega) \ll 1, O(W)=1]$ and a vortex with a small axial flow $[O(\Omega)=1, O(W) \ll 1]$.

First, when the axial flow is small such that $W=O(1 / \kappa)$, we have $\alpha=O(1 / \kappa)$, $\Phi=O(1)$ and $H=O(1 / \kappa)$. Thus, the scaling for $S$ remains $S=O(1)$ so that $B_{0}=O(1)$ but $B_{1}=O(1 / \kappa)$. Therefore, we still have $B=O(1)$ at leading order in this limit. 
Second, in the opposite limit of a slowly rotating jet such that $\Omega=O(1 / \kappa)$, we have $\alpha=\pi / 2+O(1 / \kappa), \Phi=O\left(1 / \kappa^{2}\right)$ and $H=O(1 / \kappa)$. The appropriate scaling for the Doppler shifted growth rate is then $S=O(1 / \kappa)$ implying that (3.8) becomes $\Lambda^{\prime}\left(r^{*}\right)=O\left(1 / \kappa^{2}\right)$. This limit can be treated by introducing the rescaled variables: $\hat{S}=S \kappa, \hat{\Phi}=\Phi \kappa^{2}, \hat{H}=H \kappa, \hat{\sigma}=\sigma \kappa, \hat{\Lambda}=\Lambda \kappa$ where the quantities with a hat are of order unity. Then, the functions $B_{i}$ expressed in terms of the quantities with a hat become equivalent to those when $\Omega=O(1)$ and $W=O(1)$. This limit is therefore not different from the case $\Omega=O(1)$ and $W=O(1)$ considered in $\S 3.1$ after applying the above rescaling. We conclude that the function $B$ remains also of order unity at leading order in the limit of a slowly rotating jet.

\section{Appendix B. WKB eigensolution}

In this appendix, we derive the WKB approximation of the eigenmode. The WKB approximations that decay on the real $r$-axis away from the stationary point $r^{*}$ are

$$
\psi=\frac{A_{1}}{B(r)^{1 / 4}} \exp \left(-\kappa \int_{r}^{r_{1}} \sqrt{B(u)} \mathrm{d} u\right)
$$

for $\kappa^{3 / 4}\left(r-r^{*}\right) \rightarrow-\infty$ and

$$
\psi=\frac{A_{2}}{B(r)^{1 / 4}} \exp \left(-\kappa \int_{r_{2}}^{r} \sqrt{B(u)} \mathrm{d} u\right)
$$

for $\kappa^{3 / 4}\left(r-r^{*}\right) \rightarrow+\infty$, where $A_{1}, A_{2}$ are constants and $r_{1}, r_{2}$ are the two turning points. Note that only the leading orders of the function $B$ need to be considered in (B 1)-(B 2): $B \simeq B_{0}+B_{1} / \kappa$.

The WKB approximations (B 1) and (B 2) match the local solution (3.14) in the neighbourhood of $r^{*}$ if $A_{2}=(-1)^{n} A_{1}=A(2 n+1)^{n / 2}(2 e)^{-1 / 4-n / 2}\left(-B\left(r^{*}\right) / 2\right)^{1 / 4}$ where $A$ is defined in (3.14). These approximations are actually valid in all regions of the complex plane far away from $r^{*}$ provided that continuity is ensured when crossing branch cuts (Heading 1962).

Hence, when the two turning points are sufficiently far from the real axis, the WKB solution (B 1) (or equivalently (B 2)) is uniformly valid along the real axis if continuity is preserved across the branch cuts. When the turning points $r_{1}$ and $r_{2}$ are close to the real axis, the local solution (3.14) should be used close to the turning points and (B 1) or (B 2) otherwise.

Figure 11 shows an example of comparison between the exact and the WKB eigensolutions for the Batchelor and Carton and McWilliams vortices. In both cases, the turnings points are sufficiently far from the real axis and the WKB solution (B 1) has been used everywhere along the real axis. The agreement with the exact eigensolution is very good.

\section{Appendix C. Small generalized Rayleigh discriminant}

The formula (3.25) is no longer valid when the generalized Rayleigh discriminant $\Phi\left(r_{0}\right)$ becomes small such that $\Phi\left(r_{0}\right)=O\left(1 / \kappa^{2}\right)$. Indeed, this implies that $S\left(r_{0}\right) \sim$ $\sqrt{-\Phi\left(r_{0}\right)}=O(1 / \kappa)$ so that $B_{1} / \kappa=O\left(B_{0}\right)$. Hence, the function $B_{1}$ is not negligible at leading order. With these scaling laws, the regions (3.10) and (3.12) of validity of the local solution and the WKB approximations near the stationary point $r^{*}$ become

$$
\left|r-r^{*}\right| \ll \frac{1}{\kappa \sqrt{\Lambda^{\prime \prime}\left(r^{*}\right)}},
$$


(a)

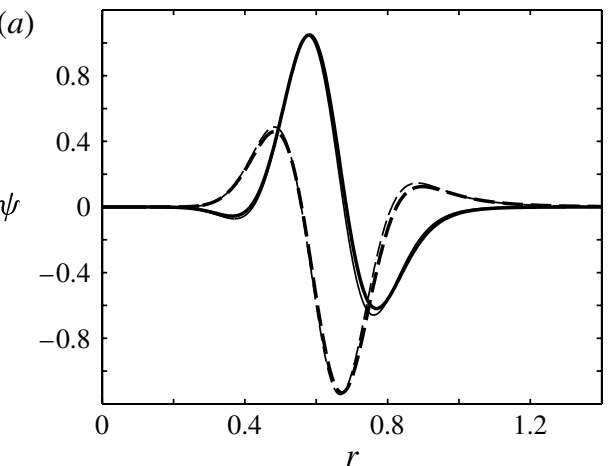

(b)

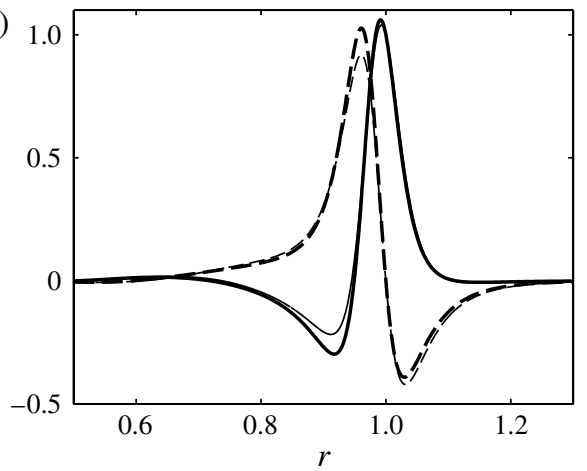

FIgURE 11. Comparison between the exact eigensolution (bold lines) and the WKB approximation (B 1) (thin lines) for (a) the Batchelor vortex for $q=0.8, m=-8, k=3$ and $(b)$ the Carton and McWilliams vortex for $\alpha=4, \gamma=2, W_{0}=0.3, m=-5, k=15$. The solid and dashed lines show the real an imaginary part, respectively.

and

$$
\left|r-r^{*}\right| \gg \frac{1}{\kappa \Lambda^{\prime \prime}\left(r^{*}\right)^{1 / 4}},
$$

respectively. Therefore, when $\Lambda^{\prime \prime}\left(r^{*}\right)=O(1)$, there is no overlap region between the local solution and the WKB solution near the stationary point so that the WKB analysis performed in $\S 3$ ceases to be valid. Nevertheless, another approach can be used as shown by Stewartson \& Capell (1985).

In contrast, when $\Lambda^{\prime \prime}\left(r^{*}\right) \ll 1$, the intervals (C 1) and (C 2) share a common overlap region and the WKB relation (3.13) remains valid. By introducing the rescaled variables $\Phi=\hat{\Phi} / \kappa^{2}, S=\hat{S} / \kappa, B_{1}=\kappa \hat{B}_{1}$, where quantities with a hat are of order unity, into (3.18), we obtain at leading order

$$
\sigma_{0}=-\mathrm{i} \kappa \Lambda\left(r_{0}\right)-\frac{H\left(r_{0}\right)}{2 \kappa f\left(r_{0}\right)}+\sqrt{-\Phi\left(r_{0}\right)+\left(\frac{H\left(r_{0}\right)}{2 \kappa f\left(r_{0}\right)}\right)^{2}},
$$

while (3.17) is again satisfied if

$$
\frac{\partial \sigma_{0}}{\partial r_{0}}=0
$$

The first-order correction for the growth rate is

$$
\delta \sigma=-\frac{2 n+1}{\kappa \sqrt{2}} \frac{\sqrt{\frac{\partial^{2} B_{0}}{\partial r^{2}}\left(r_{0}, \sigma_{0}\right)+\kappa^{-1} \frac{\partial^{2} B_{1}}{\partial r^{2}}\left(r_{0}, \sigma_{0}\right)}}{\partial B_{0}\left(r_{0}, \sigma_{0}\right) / \partial \sigma+\kappa^{-1} \partial B_{1}\left(r_{0}, \sigma_{0}\right) / \partial \sigma} .
$$

If it is assumed that $\Phi\left(r_{0}\right)=O(1)$ in the previous formulae, we recover (3.25) and (3.22). Figure 12 shows that the growth rate predicted by these formulae is in fact very close to the growth rate obtained from the implicit dispersion relation (6.2). 

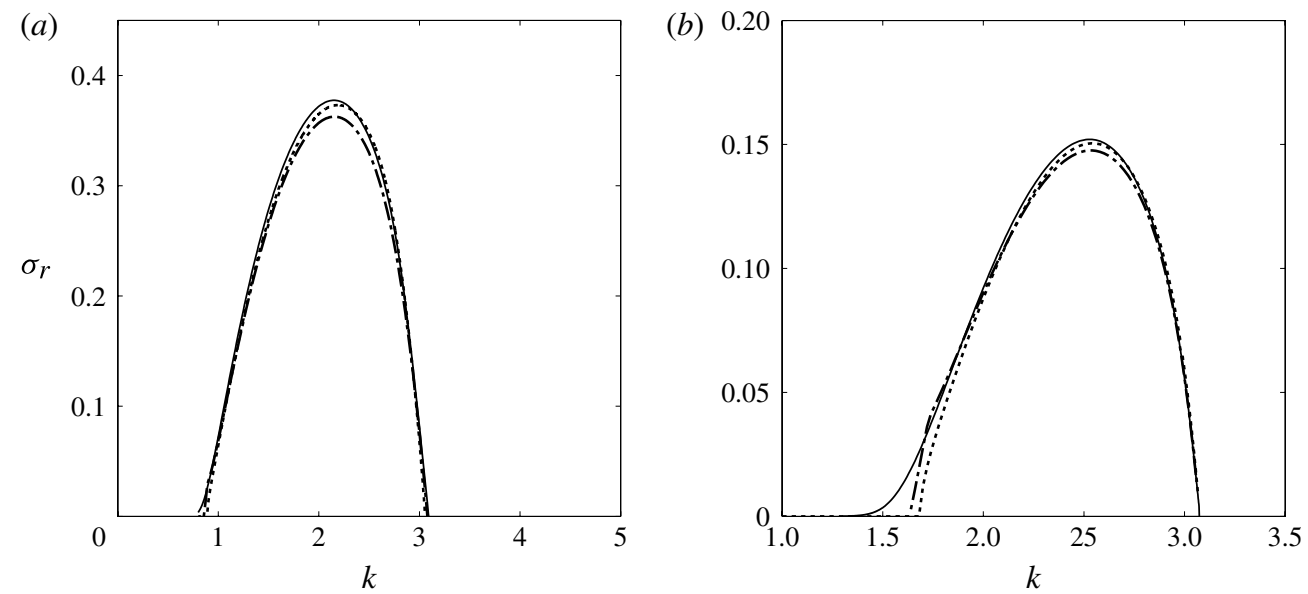

FIGURE 12. Growth rate $\sigma_{r}$ for the azimuthal wavenumber $m=-4$ for $(a) q=0.8$ and (b) $q=1.3$ as a function of the axial wavenumber $k$ for the Batchelor vortex. The solid line shows the exact growth rate, the dotted line represents (6.2) and the dash-dotted line shows the approximation derived in appendix $\mathrm{C}$.

\section{Appendix D. Asymptotic formula of Leibovich \& Stewartson (1983)}

The asymptotic growth rate obtained by Leibovich \& Stewartson (1983) for the leading unstable mode $n=0$ for the Batchelor vortex has been rederived for an arbitrary vortex following their approach. In terms of $\kappa$, the growth rate expansion reads

$$
\sigma=-\mathrm{i} \kappa \Lambda\left(r_{00}\right)+\sqrt{-\Phi\left(r_{00}\right)}+\frac{\sigma_{1}}{\kappa^{1 / 2}}+\frac{\sigma_{2}}{\kappa}+\frac{\sigma_{3}}{\kappa^{3 / 2}}+\cdots
$$

where $r_{00}$ is given by (4.2) and

$$
\begin{aligned}
\sigma_{1}= & -\frac{1}{2 \sqrt{2}}(1+\mathrm{i}) \frac{(-\Phi)^{1 / 4}}{\sqrt{f}} \sqrt{\Lambda^{\prime \prime}}, \\
\sigma_{2}= & \mathrm{i} \frac{\Phi^{\prime 2}}{8 \Phi \Lambda^{\prime \prime}}-\frac{H}{2 f}+\mathrm{i} \frac{9 \Lambda^{\prime \prime}}{32 f}, \\
\sigma_{3}= & \frac{\mathrm{i}-1}{64 \sqrt{2}(-\Phi)^{5 / 4} \Lambda^{\prime \prime 3 / 2} f^{3 / 2}} \\
& {\left[8 f \Phi^{\prime 2} \Lambda^{\prime \prime}+\Phi\left(\frac{79}{8} \Lambda^{\prime \prime 3}+16 \mathrm{i} H \Lambda^{\prime \prime 2}+8 \Phi^{\prime} f \Lambda^{\prime \prime \prime}-8 \Lambda^{\prime \prime}\left(f \Phi^{\prime}\right)^{\prime}\right)\right] . }
\end{aligned}
$$

It has not been possible to check the order $O\left(\kappa^{-3 / 2}\right)$ against the results of Leibovich \& Stewartson (1983) since some intermediate parameters at this order are not defined in their paper. However, it has been checked that the formula (D 1) gives almost the same plots as in their paper.

\section{REFERENCES}

Antkowiak, A. \& BRAncher, P. 2004 Transient energy growth for the Lamb-Oseen vortex. Phys. Fluids 16, L1-L4.

BAtchelor, G. K. 1964 Axial flow in trailing line vortices. J. Fluid Mech. 20, 645-658. 
BAYLY, B. J. 1988 Three-dimensional centrifugal-type instabilities in inviscid two-dimensional flows. Phys. Fluids 31, 56-64.

Bender, C. M. \& Orszag, S. A. 1978 Advanced Mathematical Methods for Scientists and Engineers. McGraw-Hill.

Billant, P. \& Gallaire, F. 2005 Generalized Rayleigh criterion for non-axisymmetric centrifugal instabilities. J. Fluid Mech. 542, 365-379.

Carton, X. \& McWilliams, J. C. 1989 Barotropic and baroclinic instabilities of axisymmetric vortices in a quasi-geostrophic model. In Mesoscale/Synoptic Coherent Structures in Geophysical Turbulence (ed. J. Nihoul \& B. Jamart), pp. 225-244. Elsevier.

Delbende, I., Chomaz, J.-M. \& Huerre, P. 1998 Absolute/convective instabilities in the Batchelor vortex: a numerical study of the linear impulse response. J. Fluid Mech. 355, 229-254.

Delbende, I. \& Rossi, M. 2005 Nonlinear evolution of a swirling jet instability. Phys. Fluids 17, 044103.

Di Pierro, B. \& ABID, M. 2010 Instabilities of variable-density swirling flows. Phys. Rev. E 82, 046312.

Di PIERro, B. \& ABID, M. 2012 Rayleigh-Taylor instability in variable density swirling flows. Eur. Phys. J. B 85, 1-8.

Duck, P. W. \& Foster, M. R. 1980 The inviscid stability of a trailing line vortex. Z. Angew. Math. Phys. 31, 524-532.

ECKHOFF, K. S. 1984 A note on the instability of columnar vortices. J. Fluid Mech. 145, 417-421.

Eckhoff, K. S. \& Storesletten, L. 1978 A note on the stability of steady inviscid helical gas flows. J. Fluid Mech. 89, 401-411.

EMANUEL, K. A. 1984 A note on the stability of columnar vortices. J. Fluid Mech. 145, $235-238$.

FABRE, D. \& JACQUIN, L. 2004 Viscous instabilities in trailing vortices at large swirl numbers. J. Fluid Mech. 500, 239-262.

FABRE, D. \& LE DIZÈs, S. 2008 Viscous and inviscid centre modes in the linear stability of vortices: the vicinity of the neutral curves. J. Fluid Mech. 603, 1-38.

Gallaire, F. \& Chomaz, J.-M. 2003 Mode selection in swirling jet experiments: a linear stability analysis. J. Fluid Mech. 494, 223-253.

HeAding, J. 1962 An introduction to phase-integral methods. John Wiley \& Sons.

HeAton, C. J. $2007 a$ Centre modes in inviscid swirling flows and their application to the stability of the Batchelor vortex. J. Fluid Mech. 576, 325-348.

HeAton, C. J. $2007 b$ Optimal growth of the Batchelor vortex viscous modes. J. Fluid Mech. 592, 495-505.

Heaton, C. J. \& PeAke, N. 2006 Algebraic and exponential instability of inviscid swirling flow. J. Fluid Mech. 565, 279-318.

Heaton, C. J. \& Peake, N. 2007 Transient growth in vortices with axial flow. J. Fluid Mech. 587, 271-301.

HowARD, L. N. \& GuPTA, A. S. 1962 On the hydrodynamic and hydromagnetic stability of swirling flows. J. Fluid Mech. 14, 463-476.

Khorrami, M. R. 1991 On the viscous modes of instability of a trailing line vortex. J. Fluid Mech. 225, 197-212.

LE DIZÈs, S. \& FABRE, D. 2007 Large-Reynolds-number asymptotic analysis of viscous centre modes in vortices. J. Fluid Mech. 585, 153-180.

LE Dizès, S. \& FABRE, D. 2010 Viscous ring modes in vortices with axial jet. Theor. Comput. Fluid Dyn. 24, 349-361.

Leblanc, S. \& Le Duc, A. 2005 The unstable spectrum of swirling gas flows. J. Fluid Mech. 537, $433-442$.

Leibovich, S. \& Stewartson, K. 1983 A sufficient condition for the instability of columnar vortices. J. Fluid Mech. 126, 335-356.

Lessen, M. \& Paillet, F. 1974 Stability of a trailing line vortex. Part 2. Viscous theory. J. Fluid Mech. 65, 769-779.

Lessen, M., Singh, P. J. \& Paillet, F. 1974 Stability of a trailing line vortex. Part 1. Inviscid theory. J. Fluid Mech. 63, 753-763. 
LudwIEG, H. 1960 Stabilität der strömung in einem zylindrischen ringraum. Z. Flugwiss. 8, $135-140$.

MAYeR, E. W. \& Powell, K. G. 1992 Viscous and inviscid instabilities of a trailing vortex. J. Fluid Mech. 245, 91-114.

MüLLER, S. 2007 Numerical investigations of compressible turbulent swirling jet flows. PhD thesis, Swiss Federal Institute of Technology Zürich.

Müller, S. B. \& KleISER, L. 2008 Viscous and inviscid spatial stability analysis of compressible swirling mixing layers. Phys. Fluids 20, 114103.

Olendraru, C. \& Sellier, A. 2002 Viscous effects in the absolute-convective instability of the Batchelor vortex. J. Fluid Mech. 459, 371-396.

RaYleigh, LORD 1917 On the dynamics of revolving fluids. Proc. R. Soc. Lond. A 93, 148-154.

SCHIFF, L. I. 1968 Quantum Mechanics, 3rd edn. McGraw-Hill.

Sipp, D., FABre, D., Michelin, S. \& JACQuin, L. 2005 Stability of a vortex with a heavy core. J. Fluid Mech. 526, 67-76.

SmYth, W. D. \& MCWilliams, J. C. 1998 Instability of an axisymmetric vortex in a stably stratified, rotating environment. Theor. Comput. Fluid Dyn. 11, 305-322.

StewARTSON, K. \& BROWN, S. N. 1985 Near-neutral centre-modes as inviscid perturbations to a trailing line vortex. J. Fluid Mech. 156, 387-399.

Stewartson, K. \& CAPell, K. 1985 On the stability of ring modes in a trailing line vortex - the upper neutral points. J. Fluid Mech. 156, 369-386.

STEWARTSON, K \& LEIBOVICH, S. 1987 On the stability of a columnar vortex to disturbances with large azimuthal wavenumber - the lower neutral points. J. Fluid Mech. 178, 549-566.

Sun, D.-J., Hu, G.-H., GAO, Z. \& YIN, X.-Y. 2002 Stability and temporal evolution of a swirling jet with centrifugally unstable azimuthal velocity. Phys. Fluids 14, 4081-4084.

Synge, J. L. 1933 The stability of heterogeneous liquids. Trans. R. Soc. Can. 27, 1-18. 